REPRESENTATION THEORY

An Electronic Journal of the American Mathematical Society

Volume 14, Pages 713-746 (December 1, 2010)

S $1088-4165(2010) 00387-8$

\title{
ON THE IRREDUCIBILITY OF LOCALLY ANALYTIC PRINCIPAL SERIES REPRESENTATIONS
}

\author{
SASCHA ORLIK AND MATTHIAS STRAUCH
}

\begin{abstract}
Let $\mathbf{G}$ be a $p$-adic connected reductive group with Lie algebra $\mathfrak{g}$. For a parabolic subgroup $\mathbf{P} \subset \mathbf{G}$ and a finite-dimensional locally analytic representation $V$ of a Levi subgroup of $\mathbf{P}$, we study the induced locally analytic G-representation $W=\operatorname{Ind}_{\mathbf{P}}^{\mathbf{G}}(V)$. Our result is the following criterion concerning the topological irreducibility of $W$ : If the Verma module $U(\mathfrak{g}) \otimes_{U(\mathfrak{p})} V^{\prime}$ associated to the dual representation $V^{\prime}$ is irreducible, then $W$ is topologically irreducible as well.
\end{abstract}

\section{INTRODUCTION}

One of the principal methods for constructing representations of reductive groups is to induce representations of parabolic subgroups which come by inflation from representations of Levi factors. This applies, for example, to the theory of algebraic representations as well as to the theory of smooth representations of $p$-adic reductive groups. In this paper we consider parabolically induced representations in the theory of locally analytic representations of $p$-adic reductive groups. A systematic framework to study locally analytic representations of $p$-adic groups was developed in the recent years mainly by P. Schneider and J. Teitelbaum, cf. ST1, ST2. Algebraic representations and smooth representations, as well as tensor products of these, provide first examples of such locally analytic representations, but there are many more. For instance, the representations which are locally analytically induced from representations of parabolic subgroups. Locally analytic principal series representations for $\mathrm{SL}_{2}(L), L$ being a finite extension of $\mathbb{Q}_{p}$, were already defined and studied by Y. Morita in $\mathrm{Mo}$. They were later reconsidered for the group $\mathrm{GL}_{2}$ in [ST1] (for $L=\mathbb{Q}_{p}$ ) and in [KS] (for arbitrary $L$ ).

In this paper we prove a general criterion for the irreducibility of parabolically induced locally analytic representations. In his thesis H. Frommer [Fr] studied locally analytic representations of $\mathbf{G}=\mathfrak{G}\left(\mathbb{Q}_{p}\right)$, where $\mathfrak{G}$ is a split reductive group over $\mathbb{Q}_{p}$, which are induced from finite-dimensional representations $V$ of a parabolic subgroup $\mathbf{P} \subset \mathbf{G}$. $V$ is a $K$-vector space, where $K$ is a complete discretely valued field extension of $\mathbb{Q}_{p}$. His main theorem is a criterion for the (topological) irreducibility of the induced representation $\operatorname{Ind}_{\mathbf{P}}^{\mathbf{G}}(V)$ in terms of a canonically associated Verma module. The crucial idea is to compare the structure of the dual space $\operatorname{Ind}_{\mathbf{P}}^{\mathbf{G}}(V)^{\prime}$ as

Received by the editors November 26, 2007 and, in revised form, March 16, 2010 and May 23, 2010

2010 Mathematics Subject Classification. Primary 22E50.

M.S. is partially supported by NSF grant DMS-0902103.

(C)2010 American Mathematical Society Reverts to public domain 28 years from publication 
a module over the distribution algebra $D(G, K)$, where $G \subset \mathbf{G}$ is a maximal compact subgroup, with the structure of an associated Verma module over the universal enveloping algebra $U(\mathfrak{g})$ of the Lie algebra $\mathfrak{g}$ of $\mathbf{G}$. In order to pass from $D(G, K)$ to $U(\mathfrak{g})$ one needs a technical result for their relation and Frommer only showed this for $L=\mathbb{Q}_{p}$. This is the reason for the restriction to the base field $\mathbb{Q}_{p}$ in $[\mathrm{Fr}$. Later, J. Kohlhaase [K1] proved this technical result for arbitrary finite extensions $L$ over $\mathbb{Q}_{p}$, which we use here to generalize Frommer's theorem to the case of a not necessarily split reductive group over an arbitrary extension of $\mathbb{Q}_{p}$.

In order to state our main result let $\mathfrak{G}$ be a connected reductive group over $L$, and let $\mathfrak{P} \subset \mathfrak{G}$ be a parabolic subgroup. We consider a locally analytic representation $V$ of the group $\mathbf{P}=\mathfrak{P}(L)$ which comes by inflation from a Levi subgroup and put $\mathbf{G}=\mathfrak{G}(L)$. Here $V$ is a vector space over a complete discretely valued extension $K$ which contains $L$ and whose absolute value induces the given absolute value on $L$. Then we have:

Main result: Suppose $\operatorname{dim}_{K}(V)<\infty$. Then the induced locally analytic representation $\operatorname{Ind}_{\mathbf{P}}^{\mathbf{G}}(V)$ is topologically irreducible if $U(\mathfrak{g}) \otimes_{U(\mathfrak{p})} V^{\prime}$ is irreducible as a module over the universal enveloping algebra $U(\mathfrak{g})$.

Our overall strategy of proof follows basically Frommer's treatment. However, we found that an essential argument in $[\mathrm{Fr}$, stating that certain distribution algebras are integral domains, is not obvious, as it is claimed there. We prove this in sections 3.3 and 5 .

But beside the fact that it is desirable to have such a criterion for the irreducibility in general, a motivation was provided by the concrete example of certain $\mathbb{Q}_{p}$-analytic principal series representations of $\mathrm{GL}_{2}\left(\mathbb{Q}_{p^{2}}\right)$ (regarded as a group over $\left.\mathbb{Q}_{p}\right)$. The interest in these representations comes from conjectural relations to two-dimensional crystalline representations of $\operatorname{Gal}\left(\overline{\mathbb{Q}}_{p} \mid \mathbb{Q}_{p^{2}}\right)$. Such induced locally analytic representations play an important role in the $p$-adic Langlands program, cf. [BS.

In the last section we consider a particular example, namely the case where $\mathfrak{G}$ comes by restriction of scalars from a group which is split over $L$. This includes, in particular, the above-mentioned case of the group $\mathrm{GL}_{2}\left(\mathbb{Q}_{p^{2}}\right)$ (regarded as a group over $\mathbb{Q}_{p}$ ).

Notation. We let $p$ be a prime number and denote by $L$ a finite extension of $\mathbb{Q}_{p}$. The normalized $p$-adic (logarithmic) valuation is denoted by $v_{p}$ (i.e. $v_{p}(p)=1$ ). We let $K \subset \mathbb{C}_{p}$ be a complete discretely valued field extension of $L$, whose absolute value $|\cdot|_{K}$ is normalized so that $|p|_{K}=p^{-1}$. The rings of integers are denoted by $\mathfrak{o}_{L}$ and $\mathfrak{o}_{K}$, respectively. For notions and notation in the context of non-archimedean functional analysis we refer to $[\underline{\mathrm{S}}$.

\section{Distribution algebras and LOCALly ANALytic RePRESENTATIONS}

2.1. Distribution algebras. In this section we recall some definitions and results about algebras of distributions attached to locally analytic groups, cf. [ST1, [ST2]. We consider a locally $L$-analytic group $H$ and denote by

$$
C^{a n}(H, K)=C_{L}^{a n}(H, K)
$$


the locally convex $K$-vector space of locally $L$-analytic functions on $H$ as defined in [ST1. The strong dual

$$
D(H, K)=D_{L}(H, K):=\left(C_{L}^{a n}(H, K)\right)_{b}^{\prime}
$$

is the topological algebra of $K$-valued distributions on $H$. Furthermore, if $H$ is compact, then $D(H, K)$ has the structure of a Fréchet algebra. The multiplication $\delta_{1} * \delta_{2}$ of distributions $\delta_{1}, \delta_{2} \in D(H, K)$ is defined by

$$
\delta_{1} * \delta_{2}(f)=\left(\delta_{1} \otimes \delta_{2}\right)\left(\left(h_{1}, h_{2}\right) \mapsto f\left(h_{1} h_{2}\right)\right),
$$

where the distribution $\delta_{1} \otimes \delta_{2} \in D(H \times H, K)$ has the property that for functions $f_{1}, f_{2} \in C^{a n}(H, K)$, one has

$$
\left(\delta_{1} \otimes \delta_{2}\right)\left(\left(h_{1}, h_{2}\right) \mapsto f_{1}\left(h_{1}\right) f_{2}\left(h_{2}\right)\right)=\delta_{1}\left(f_{1}\right) \delta_{2}\left(f_{2}\right) .
$$

The universal enveloping algebra $U(\mathfrak{h})$ of the Lie algebra $\mathfrak{h}=\operatorname{Lie}(H)$ of $H$ acts naturally on $C^{a n}(H, K)$. On elements $\mathfrak{x} \in \mathfrak{h}$, this action is given by

$$
(\mathfrak{x} f)(h)=\left.\frac{d}{d t}(t \mapsto f(\exp (-t \mathfrak{x}) h))\right|_{t=0} .
$$

This gives rise to an embedding of $U(\mathfrak{h})_{K}:=U(\mathfrak{h}) \otimes_{L} K$ into $D(H, K)$ :

$$
U(\mathfrak{h})_{K} \hookrightarrow D(H, K), \mathfrak{z} \mapsto(f \mapsto(\mathfrak{z} f)(1)) .
$$

Here $\mathfrak{z} \mapsto \dot{\mathfrak{z}}$ is the unique $K$-linear anti-automorphism of $U(\mathfrak{h})_{K}$ which induces multiplication by -1 on $\mathfrak{h}$.

\subsection{Norms and completions of distribution algebras.}

2.2.1. $p$-valuations and global charts. Let $H$ be a compact locally $\mathbb{Q}_{p}$-analytic group. Recall that a map

$$
\omega: H-\{1\} \longrightarrow\left(\frac{1}{p-1}, \infty\right) \subset \mathbb{R}
$$

is called a p-valuation (cf. L, III.2.1.2) if the following conditions hold for all $g, h \in H$ :

i) $\omega\left(g h^{-1}\right) \geq \min \{\omega(g), \omega(h)\}$,

ii) $\omega\left(g^{-1} h^{-1} g h\right) \geq \omega(g)+\omega(h)$,

iii) $\omega\left(g^{p}\right)=\omega(g)+1$.

As usual one puts $\omega(1)=\infty$ and interprets the above inequalities in the obvious sense, if a term $\omega(1)$ occurs. Let $\omega$ be a $p$-valuation on $H$. The above conditions imply that for any $\nu>0$ the sets

$$
H_{\nu}=\{h \in H \mid \omega(h) \geq \nu\} \text { and } H_{\nu^{+}}=\{g \in H \mid \omega(g)>\nu\}
$$

are normal subgroups of $H$. We put

$$
\operatorname{gr}(H)=\bigoplus_{\nu>0} H_{\nu} / H_{\nu^{+}} .
$$

The commutator induces a Lie bracket on $\operatorname{gr}(H)$ which gives $\operatorname{gr}(H)$ the structure of a Lie algebra over $\mathbb{F}_{p}$. The map $\epsilon$ defined by

$$
\epsilon: \operatorname{gr}(H) \rightarrow \operatorname{gr}(H), \epsilon\left(g H_{\nu^{+}}\right)=g^{p} H_{(\nu+1)^{+}}
$$

is an $\mathbb{F}_{p}$-linear map on $\operatorname{gr}(H)$, which gives $\operatorname{gr}(H)$ the structure of a graded Lie algebra over $\mathbb{F}_{p}[\epsilon]$, cf. $\left[\mathrm{L}\right.$, III.2.1.1. It is a free $\mathbb{F}_{p}[\epsilon]$-module, whose rank is equal to the dimension of $H$ as a $\mathbb{Q}_{p}$-analytic group, cf. loc. cit., III.3.1.3/7/9. 
If $\left(h_{1} H_{\omega\left(h_{1}\right)^{+}}, \ldots, h_{d} H_{\omega\left(h_{d}\right)^{+}}\right)$is a basis of $\operatorname{gr}(H)$ over $\mathbb{F}_{p}[\epsilon]$, then the elements $h_{1}, \ldots, h_{d}$ form a topological generating system of $H$, and the following map

$$
\mathbb{Z}_{p}^{d} \rightarrow H,\left(a_{1}, \ldots, a_{d}\right) \mapsto h_{1}^{a_{1}} \cdot \ldots \cdot h_{d}^{a_{d}}
$$

is well defined and is a homeomorphism. Moreover,

$$
\omega\left(h_{1}^{a_{1}} \cdot \ldots \cdot h_{d}^{a_{d}}\right)=\min \left\{\omega\left(h_{i}\right)+v_{p}\left(a_{i}\right) \mid i=1, \ldots, d\right\} .
$$

The sequence $\left(h_{1}, \ldots, h_{d}\right)$ is called a $p$-basis of $H$.

2.2.2. Uniform pro-p groups. We recall some definitions and results about pro- $p$ groups, cf. DDMS, ch. 3, 4. In this section $H$ will be a pro- $p$ group which is equipped with its topology as a pro-finite group. Then $H$ is called powerful if $p$ is odd (resp. $p=2$ ) and $H / \overline{H^{p}}$ (resp. $H / \overline{H^{4}}$ if $p=2$ ) is abelian. Here, $\overline{H^{p}}$ (resp. $\overline{H^{4}}$ ) is the closure of the subgroup generated by the $p$-th (resp. fourth) powers of its elements. If $H$ is topologically finitely generated, one can show that the subgroups $H^{p}$ (resp. $H^{4}$ ) are open and hence automatically closed. The lower p-series $\left(P_{i}(H)\right)_{i \geq 1}$ of an arbitrary pro- $p$ group $H$ is defined inductively by

$$
P_{1}(H)=H, P_{i+1}(H)=\overline{P_{i}(H)^{p}\left[P_{i}(H), H\right]} .
$$

If $H$ is topologically finitely generated, then the groups $P_{i}(H)$ are all open in $H$ and form a fundamental system of neighborhoods of 1 , cf. Proposition 1.16 in loc.cit. A pro- $p$ group $H$ is called uniform if it is topologically finitely generated, powerful and its lower $p$-series satisfies

$$
\left(H: P_{2}(H)\right)=\left(P_{i}(H): P_{i+1}(H)\right)
$$

for all $i \geq 1$. If $H$ is a topologically finitely generated powerful pro- $p$ group, then $P_{i}(H)$ is a uniform pro- $p$ group for all sufficiently large $i$, cf. loc.cit. 4.2. Moreover, any compact $\mathbb{Q}_{p}$-analytic group contains an open normal uniform pro- $p$ subgroup, cf. loc.cit. 8.34 .

2.2.3. The canonical p-valuation on uniform groups. Let $H$ be a uniform pro- $p$ group. It carries a distinguished $p$-valuation $\omega^{\text {can }}$ which is associated to the lower $p$-series and which we call the canonical p-valuation. In order to define it, we let $\varepsilon_{p}=2$ if $p=2$ and $\varepsilon_{p}=1$ for odd $p$. For $h \neq 1$, we then put

$$
\omega^{\text {can }}(h)=-1+\varepsilon_{p}+\max \left\{i \geq 1 \mid h \in P_{i}(H)\right\} .
$$

To verify that this gives indeed a $p$-valuation one makes use of the fact that $\left[P_{i}(H), P_{j}(H)\right] \subset P_{i+j}(H)$ for all $i, j \geq 1$, cf. Proposition 1.16 in DDMS. Also, property (iii) follows from Proposition 2.7 in loc.cit. For $p=2$ one has to use the stronger statement that $\left[P_{i}(H), P_{j}(H)\right] \subset P_{i+j+1}(H)$ for all $i, j \geq 1$, cf. Sch, proof of Proposition 2.1.

We remark that a uniform pro- $p$ group $H$ has the property that the exponential map $\exp _{H}: \operatorname{Lie}(H) \rightarrow H$, which is, for a general $p$-adic Lie group, only defined on a sufficienly small lattice in $\operatorname{Lie}(H)$, is for uniform pro- $p$ groups defined on a unique $\mathbb{Z}_{p}$-lattice $\Lambda \subset \operatorname{Lie}(H)$, which it maps bijectively onto $H$ (cf. DDMS, sec. 4.5 and sec. 9.4).

Lemma 2.2.4. (i) Any compact locally L-analytic group $G$ has a normal open subgroup $H$ with the following properties:

(1) $H$ is uniform pro-p. 
(2) Let $\Lambda \subset \operatorname{Lie}(H)$ be the $\mathbb{Z}_{p}$-lattice which the exponential map $\exp _{H}: \operatorname{Lie}(H) \rightarrow$ $H$ maps bijectively onto $H$; then $\Lambda$ is stable under multiplication by $\mathfrak{o}_{L}$, i.e., $\Lambda$ is an $\mathfrak{o}_{L}$-submodule of $\operatorname{Lie}(H)$.

(ii) Assume $H$ is uniform pro-p, and let $\Lambda$ be as in (2) above. Then, for any $\mathbb{Z}_{p}$-basis $\left(\mathfrak{x}_{1}, \ldots, \mathfrak{x}_{N}\right)$ of $\Lambda$ the map

$$
\mathbb{Z}_{p}^{N} \rightarrow H, \quad\left(a_{i}\right)_{i} \mapsto \exp _{H}\left(\mathfrak{x}_{1}\right)^{a_{1}} \cdot \ldots \cdot \exp _{H}\left(\mathfrak{x}_{N}\right)^{a_{N}}
$$

is a homeomorphism.

Proof. (i) Let $\Lambda^{\prime} \subset \operatorname{Lie}(G)$ be any $\mathfrak{o}_{L}$-lattice which is stable under the adjoint action of $G$ on $\operatorname{Lie}(G)$. For $t \gg 0$, we have that $\left[p^{t} \Lambda^{\prime}, p^{t} \Lambda^{\prime}\right] \subset p^{\varepsilon_{p}} p^{t} \Lambda^{\prime}$. Hence $p^{t} \Lambda^{\prime}$ is a uniform Lie algebra over $\mathbb{Z}_{p}$ in the sense of DDMS, sec. 9.4. For $t \gg 0$ the exponential function $\exp _{G}$ will be defined on $p^{t} \Lambda^{\prime}$, and will map $p^{t} \Lambda^{\prime}$ bijectively onto $H:=\exp _{G}\left(p^{t} \Lambda^{\prime}\right)$. It follows that $H$ is then a uniform pro- $p$ group, cf. DDMS, Theorem 9.10. $H$ is normal in $G$ because $\Lambda$ is invariant under the adjoint action of G.

(ii) By DDMS, Theorem 9.10, the $\mathbb{Z}_{p}$-Lie algebra $L_{H}$ of $H$, as defined in DDMS, sec. 4.5 , is uniform. Denote by $*$ the multiplication on $L_{H}$ as defined in DDMS, sec. 9.4, using the Baker-Campbell-Hausdorff series (which converges and is analytic on $L_{H}$, by DDMS, Lemma 9.12). Then $\left(L_{H}, *\right)$ is actually isomorphic to $H$, DDMS, Theorem 9.10. By [DDMS, Theorem 9.8, for any $\mathbb{Z}_{p}$-basis $\left(\mathfrak{x}_{i}\right)$ of $L_{H}$ the corresponding elements of $H$ will be a topological generating set.

Remark 2.2.5. (i) Pro- $p$ groups $H$ which satisfy the the properties (1) and (2) of Lemma 2.2.4 will be considered repeatedly in this paper, and, for the purpose of this paper, we will call them $L$-uniform. $L$-uniform groups are exactly the groups that satisfy condition (L) in $[\mathrm{Sch}$, , (cf. before Cor. 4.4 of [Sch]), which is in turn equivalent to the conditions in Proposition 1.3.5 of [K1]. We thought about using condition $(\mathrm{L})$ as in $\mathrm{Sch}$, but found that the formulation above is slightly more natural.

(ii) Let $H$ be a locally $L$-analytic uniform pro- $p$ group. Then the $\mathbb{Z}_{p}$-Lie algebra $L_{H}$ of $H$ in the sense of [DDMS], sec. 4.5, is not necessarily an $\mathfrak{o}_{L}$-module. Even though one has an embedding $L_{H} \hookrightarrow \operatorname{Lie}(H)$, and $\operatorname{Lie}(H)$ is an $L$-vector space, the $\mathbb{Z}_{p}$-submodule $L_{H}$ need not be stable under multiplication by $\mathfrak{o}_{L}$. For a simple example, consider the abelian group $H=\mathbb{Z}_{p}+p \mathfrak{o}_{L}$. The $\mathbb{Z}_{p}$-Lie algebra $L_{H}$ of $H$ is $H$ itself, but $L_{H}$ is not an $\mathfrak{o}_{L}$-module if $L \neq \mathbb{Q}_{p}$.

2.2.6. Norms induced by p-valuations. In this section we let $H$ be a compact $L$ analytic group. The distribution algebra $D(H, K)$ is then a Fréchet-Stein algebra in the sense of [ST2, sec. 3. This means, in particular, that there exists a family of norms \|\|$_{r}, \frac{1}{p}<r<1$, on $D(H, K)$ such that, if $D_{r}(H, K)$ denotes the completion of $D(H, K)$ with respect to \|\|$_{r}$, the convolution product $*$ on $D(H, K)$ extends by continuity to a product on $D_{r}(H, K)$. We recall briefly the construction of such a family of norms. This is done in three steps, cf. ST2 (proof of Theorem 5.1).

Step 1. We denote by $R_{\mathbb{Q}_{p}}^{L} H$ the group $H$ when considered as a locally $\mathbb{Q}_{p^{-}}$ analytic group, and we put $d=\operatorname{dim}\left(R_{\mathbb{Q}_{p}}^{L} H\right)$. Let $H_{0} \subset R_{\mathbb{Q}_{p}}^{L} H$ be an open normal subgroup which is equipped with a $p$-valuation $\omega$. For instance, by [DDMS], 8.34, we may choose $H_{0}$ to be an open normal uniform pro- $p$ subgroup, and we may take for $\omega$ the canonical valuation associated to its lower $p$-series (cf. 2.2.3). Then we consider the graded group $\operatorname{gr}\left(H_{0}\right)$ (which depends on $\omega$ ). A choice of a basis 
of $\operatorname{gr}\left(H_{0}\right)$ gives rise to a homeomorphism $\psi: \mathbb{Z}_{p}^{d} \rightarrow H_{0}$ (cf. 2.2.1), which in turn induces an isomorphism of locally convex $K$-vector spaces

$$
\psi^{*}: C^{a n}\left(H_{0}, K\right) \stackrel{\simeq}{\longrightarrow} C^{a n}\left(\mathbb{Z}_{p}^{d}, K\right)
$$

as well as an isomorphism of $K$-Banach spaces of continuous functions

$$
\psi^{*}: C\left(H_{0}, K\right) \stackrel{\simeq}{\longrightarrow} C\left(\mathbb{Z}_{p}^{d}, K\right) .
$$

Using Mahler expansions ([L], III.1.2.4) we can express elements of $C\left(\mathbb{Z}_{p}^{d}, K\right)$ as the series

$$
f(x)=\sum_{n \in \mathbb{N}_{0}^{d}} c_{n}\left(\begin{array}{l}
x \\
n
\end{array}\right)
$$

where $c_{n} \in K$ and

$$
\left(\begin{array}{l}
x \\
n
\end{array}\right):=\left(\begin{array}{l}
x_{1} \\
n_{1}
\end{array}\right) \cdot \ldots \cdot\left(\begin{array}{l}
x_{d} \\
n_{d}
\end{array}\right)
$$

for the multi-indices $x=\left(x_{1}, \ldots, x_{d}\right)$ and $n=\left(n_{1}, \ldots, n_{d}\right) \in \mathbb{N}_{0}^{d}$. Further, we have $\left|c_{n}\right| \rightarrow 0$ as $|n|=n_{1}+\ldots+n_{d} \rightarrow \infty$. A continuous function $f \in C\left(\mathbb{Z}_{p}^{d}, K\right)$ is locally analytic if and only if $\left|c_{n}\right| r^{|n|} \rightarrow 0$ for some $r>1$. Consider the group algebra $K\left[H_{0}\right]$ of $H_{0}$. By identifying elements of $H_{0}$ with Dirac distributions, we get an embedding

$$
K\left[H_{0}\right] \hookrightarrow D\left(H_{0}, K\right) .
$$

Write $b_{i}:=h_{i}-1 \in K\left[H_{0}\right]$ and set for $n \in \mathbb{N}_{0}^{d}$,

$$
\mathbf{b}^{n}=b_{1}^{n_{1}} \cdots b_{d}^{n_{d}} .
$$

Then we have $\mathbf{b}^{n}(f)=c_{n}$ for any continuous function $f \in C\left(H_{0}, K\right)$, where the $c_{n}$ are the Mahler coefficients of $\psi^{*}(f) \in C\left(\mathbb{Z}_{p}^{d}, K\right)$. It follows that every distribution $\lambda \in D\left(H_{0}, K\right)$ has the shape

$$
\lambda=\sum_{n \in \mathbb{N}_{0}^{d}} d_{n} \mathbf{b}^{n}
$$

where $\left\{d_{n} r^{|n|} \mid n \in \mathbb{N}_{0}^{d}\right\}$ is a bounded set for all $0<r<1$. The norm $\|\cdot\|_{r}$ on $D\left(H_{0}, K\right)$ is then defined by

$$
\left\|\sum_{n \in \mathbb{N}_{0}^{d}} d_{n} \mathbf{b}^{n}\right\|_{r}=\sup \left\{\left|d_{n}\right| r^{\tau(n)} \mid n \in \mathbb{N}_{0}^{d}\right\} .
$$

Here, $\tau(n)$ is given by $\tau(n)=\sum_{i} n_{i} \omega\left(h_{i}\right)$. The Banach algebra $D_{r}\left(H_{0}, K\right)$ is defined to be the completion of $D\left(H_{0}, K\right)$ with respect to $\|\cdot\|_{r}$. Thus we obtain

$$
D_{r}\left(H_{0}, K\right)=\left\{\sum_{n \in \mathbb{N}_{0}^{d}} d_{n} \mathbf{b}^{n} \in K\left[\left[b_{1}, \ldots, b_{d}\right]\right]\left|\lim _{|n| \rightarrow \infty}\right| d_{n} \mid r^{\tau(n)}=0\right\} .
$$

Furthermore, for $\frac{1}{p}<r<1$, the norm $\|\cdot\|_{r}$ is multiplicative (cf. [ST2], Theorem 4.5) and does not depend on the chosen basis (loc. cit., before Theorem 4.11). We obtain a projective system of noetherian Banach algebras such that

$$
D\left(H_{0}, K\right)=\lim _{r} D_{r}\left(H_{0}, K\right) .
$$

Moreover, the transition maps

$$
D_{r^{\prime}}\left(H_{0}, K\right) \rightarrow D_{r}\left(H_{0}, K\right)
$$

are flat for $\frac{1}{p}<r \leq r^{\prime}<1$.

Step 2. We extend the norm $\|\cdot\|_{r}$ on $D\left(H_{0}, K\right)$ to a norm on $D\left(R_{\mathbb{Q}_{p}}^{L} H, K\right)$ as follows. Let $\eta_{1}, \ldots, \eta_{s}$ be a system of coset representatives of $H / H_{0}$. Then 
the Dirac distributions $\delta_{\eta_{1}}, \ldots, \delta_{\eta_{s}}$ form a basis of $D\left(R_{\mathbb{Q}_{p}}^{L} H, K\right)$ over $D\left(H_{0}, K\right)$. Writing $\mu \in D\left(R_{\mathbb{Q}_{p}}^{L} H, K\right)$ as $\mu=\lambda_{1} \delta_{\eta_{1}}+\ldots+\lambda_{s} \delta_{\eta_{s}}$, we put

$$
q_{r}(\mu)=\max \left\{\left\|\lambda_{i}\right\|_{r} \mid 1 \leq i \leq s\right\} .
$$

Again, we obtain a system of sub-multiplicative norms $q_{r}, \frac{1}{p}<r<1$, and a projective system $D_{r}\left(R_{\mathbb{Q}_{p}}^{L} H, K\right)$ of $K$-Banach algebras fulfilling the conditions above, and we have $D\left(R_{\mathbb{Q}_{p}}^{L} H, K\right)=\lim _{\longleftarrow} D_{r}\left(R_{\mathbb{Q}_{p}}^{L} H, K\right)$. The norms $q_{r}$ do not depend on the chosen representatives. It is worth noting at this point that the norms $q_{r}$ are in general not multiplicative, because $H$ may contain non-trivial elements of finite order which cause the group ring, as well as the rings of distribution, to have non-trivial zero divisors 1 .

Step 3. Locally $L$-analytic functions on $H$ are obviously locally $\mathbb{Q}_{p}$-analytic functions on $R_{\mathbb{Q}_{p}}^{L} H$, and hence there is a canonical map

$$
C_{L}^{a n}(H, K) \hookrightarrow C_{\mathbb{Q}_{p}}^{a n}\left(R_{\mathbb{Q}_{p}}^{L} H, K\right)
$$

which is a closed embedding (cf. the proof of Theorem 5.1 in [ST2]). This map induces by duality a continuous surjection

$$
D_{\mathbb{Q}_{p}}\left(R_{\mathbb{Q}_{p}}^{L} H, K\right) \rightarrow D_{L}(H, K)
$$

on the distribution algebras. We denote the induced residue norm on $D_{L}(H, K)$ respectively on the completion $D_{r}(H, K)$ by $\bar{q}_{r}$. Once again, we have $D_{L}(H, K)=$ $\lim _{\longleftarrow} D_{r}(H, K)$.

Although we will mostly consider the case where the parameter $r$ lies strictly between $\frac{1}{p}$ and 1 , we point out that the norms $\bar{q}_{r}$ are defined, as norms of $K$-vector spaces, for any $r \in(0,1)$. This is used later in the proof of Proposition 3.4.10, cf. Lemma 3.4.9

Remark 2.2.7. We keep the notation from the preceding paragraph. In the recipe for the norm $\bar{q}_{r}$ on $D_{L}(H, K)$ given above, we could have carried out steps two and three in reverse order with the same resulting norm. To be precise, let $\overline{\|}_{r}$ be the quotient norm on $D_{L}\left(H_{0}, K\right)$ induced by the surjection $D_{\mathbb{Q}_{p}}\left(R_{\mathbb{Q}_{p}}^{L} H_{0}, K\right) \rightarrow$ $D_{L}\left(H_{0}, K\right)$. Again, we have

$$
D_{L}(H, K)=\bigoplus_{i=1}^{s} D_{L}\left(H_{0}, K\right) * \delta_{\eta_{i}}
$$

and can hence consider the maximum norm $\hat{q}_{r}$ on $D_{L}(H, K)$ induced from this decomposition and the norm $\overline{\| \cdot}_{r}$ on $D_{L}\left(H_{0}, K\right)$. Then it is not difficult to check that $\hat{q}_{r}$ coincides with the norm $\bar{q}_{r}$ defined above, cf. [Sch], Lemma 4.4.

\footnotetext{
${ }^{1} \mathrm{In}[\mathrm{Fr}]$ it is mistakenly stated that these norms were multiplicative so that the corresponding completions would be integral domains. As a consequence, some completed distribution algebras were claimed to be obviously integral domains.
} 


\subsection{The closure of the enveloping algebra.}

2.3.1. Definition of $U_{r}\left(\mathfrak{h}, H_{0}\right)$. Let $H$ be a compact locally $L$-analytic group and let $H_{0} \subset H$ be an open normal subgroup, which is equipped with a $p$-valuation $\omega$. Associated to $\omega$ there is a norm $\bar{q}_{r}$ on $D(H, K)$ as defined in 2.2.6. We write

$$
U_{r}\left(\mathfrak{h}, H_{0}\right) \subset D_{r}\left(H_{0}, K\right)
$$

for the topological closure of $U(\mathfrak{h}) \otimes_{L} K$ in $D_{r}\left(H_{0}, K\right)$. The following theorem by Kohlhaase generalizes a result of Frommer who considered the case of $\mathbb{Q}_{p}$-analytic groups. It is this basic technical result which we use to generalize Frommer's irreducibility criterion.

Theorem 2.3.2 (ㅈ1, 1.4.2). Let $H$ be a compact locally L-analytic group of dimension d with Lie algebra $\mathfrak{h}=\operatorname{Lie}(H)$ and $r \in p^{\mathbb{Q}}$ with $\frac{1}{p}<r<1$.

(i) Let $H_{0} \subset H$ be a normal open subgroup which is L-uniform (cf. Remark 2.2.5). Then, if we equip $R_{\mathbb{Q}_{p}}^{L} H_{0}$ with its canonical valuation (2.2.3), $D_{r}\left(H_{0}, K\right)$ is a free, finitely generated module over the noetherian subalgebra $U_{r}\left(\mathfrak{h}, H_{0}\right)$.

(ii) Let the normal L-uniform subgroup $H_{0}=\exp _{H}(\Lambda) \subset H$ be as above, and let $\mathfrak{X}=\left(\mathfrak{X}_{1}, \ldots, \mathfrak{X}_{d}\right)$ be an $\mathfrak{o}_{L}$-basis of $\Lambda$. Then there is a norm $\nu_{r}$ on $U_{r}\left(\mathfrak{h}, H_{0}\right)$ which is equivalent to $\bar{q}_{r}$, such that $U_{r}\left(\mathfrak{h}, H_{0}\right)$ consists of exactly those series

$$
\sum_{n \in \mathbb{N}_{0}^{d}} d_{n} \mathfrak{X}^{n}
$$

for which

$$
\lim _{|n| \rightarrow \infty}\left|d_{n}\right| \nu_{r}\left(\mathfrak{X}^{n}\right)=0,
$$

and $\nu_{r}\left(\sum_{n} d_{n} \mathfrak{X}^{n}\right)=\sup _{n}\left|d_{n}\right| \nu_{r}\left(\mathfrak{X}^{n}\right)$.

Remark 2.3.3. In [K1, 1.4.2, the conditions imposed on $H_{0}$ are those of [K1, 1.3.5. However, a group $H_{0}$ which is $L$-uniform automatically satisfies the conditions of K1], 1.3.5 2

Corollary 2.3.4. Let $H$ be a compact locally L-analytic group, and let $H_{0} \subset H$ be an open normal subgroup as in Theorem $2.3 .2(\mathrm{i})$. Suppose that $D_{r}(H, K)$ is an integral domain. Then any non-zero left ideal $I$ of $D_{r}(H, K)$ has non-zero intersection with $U_{r}\left(\mathfrak{h}, H_{0}\right)$.

Proof. Compare $\mathrm{Fr}$, Corollary 5. Since $H$ is compact, $D_{r}(H, K)$ is a finite $D_{r}\left(H_{0}, K\right)$-module, and so $D_{r}(H, K)$ is finite over $U_{r}\left(\mathfrak{h}, H_{0}\right)$. Therefore, there exists for any $F \in D_{r}(H, K)$, a polynomial $P=\sum_{i=0}^{N} a_{i} X^{i}, a_{i} \in U_{r}\left(\mathfrak{h}, H_{0}\right)$ with $P(F)=0$. Because we assumed $D_{r}(H, K)$ to be an integral domain, we may find a polynomial with $a_{0} \neq 0$. So, if $F \in I$, then

$$
0 \neq a_{0}=-\sum_{i=1}^{N} a_{i} F^{i} \in I \cap U_{r}\left(\mathfrak{h}, H_{0}\right) .
$$

\footnotetext{
${ }^{2}$ On the other hand, one can show that a group satisfying the conditions of [K1], 1.3.5, is actually $L$-uniform. We will not need this fact in this paper.
} 
2.4. Locally analytic representations. We conclude this section by recalling some facts of locally analytic representations. Let $H$ be a locally $L$-analytic group, $V$ a Hausdorff locally convex $K$-vector space, and $\rho: H \rightarrow \mathrm{GL}_{K}(V)$ a homomorphism. Then $\rho$ (or the pair $(V, \rho)$ ) is called a locally analytic representation of $H$ if the topological $K$-vector space $V$ is barrelled, each $h \in H$ acts $K$-linearly and continuously on $V$, and the orbit maps $\rho_{v}: H \rightarrow V, h \mapsto \rho(h)(v)$, are locally analytic maps for all $v \in V$, cf. [ST1, sec. 3. If $V$ is of compact type, i.e., a compact inductive limit of Banach spaces, the strong dual $V_{b}^{\prime}$ is a nuclear Fréchet space and a separately continuous left $D(H, K)$-module. The module structure is given as follows:

$$
D(H, K) \otimes_{K} V_{b}^{\prime} \rightarrow V_{b}^{\prime}, \delta \otimes \varphi \mapsto\left(v \mapsto \delta\left(g \mapsto \varphi\left(\rho\left(g^{-1}\right) v\right)\right)\right) .
$$

This functor gives an equivalence of categories:

$$
\left\{\begin{array}{c}
\text { locally analytic } H \text {-represen- } \\
\text { tations on } K \text {-vector spaces } \\
\text { of compact type with } \\
\text { continuous linear } H \text {-maps }
\end{array}\right\} \rightarrow\left\{\begin{array}{c}
\text { separately continuous } D(H, K)- \\
\text { modules on nuclear Fréchet spa- } \\
\text { ces with continuous } D(H, K)- \\
\text { module maps }
\end{array}\right\} .
$$

In particular, $V$ is topologically irreducible if $V_{b}^{\prime}$ is a simple $D(H, K)$-module.

For any closed subgroup $H^{\prime}$ of $H$ and any locally analytic representation $V$ of $H^{\prime}$, we denote by $\operatorname{Ind}_{H^{\prime}}^{H}(V)$ the induced locally analytic representation. We recall the definition

$$
\operatorname{Ind}_{H^{\prime}}^{H}(V):=\left\{f \in C^{a n}(H, V) \mid f\left(h^{\prime} \cdot h\right)=h^{\prime} \cdot f(h) \forall h^{\prime} \in H^{\prime}, \forall h \in H\right\} .
$$

The group $H$ acts on this vector space by $(h \cdot f)(x)=f(x h)$. We have a Frobenius reciprocity in the category of locally analytic representations (see $\mathrm{Fe}$ Theorem 4.2.6):

$$
\operatorname{Hom}_{H}^{\text {cont }}\left(W, \operatorname{Ind}_{H^{\prime}}^{H} V\right) \cong \operatorname{Hom}_{H^{\prime}}^{\text {cont }}\left(\operatorname{Res}_{H^{\prime}}^{H} W, V\right) .
$$

Here, $\operatorname{Res}_{H^{\prime}}^{H}(W)$ denotes as usual the restriction, viewing $W$ via the embedding $H^{\prime} \hookrightarrow H$ as a $H^{\prime}$-representation. Consider the canonical map of $D(H, K)$-modules

$$
D(H, K) \otimes_{D\left(H^{\prime}, K\right)} V^{\prime} \rightarrow\left(\operatorname{Ind}_{H^{\prime}}^{H} V\right)_{b}^{\prime}, \delta \otimes \varphi \mapsto \delta \cdot \varphi,
$$

with $(\delta \cdot \varphi)(f)=\delta\left(g \mapsto \varphi\left(f\left(g^{-1}\right)\right)\right)$. Suppose $H=C \cdot H^{\prime}$ with a compact subgroup $C \subset H$, such that $C \cap H^{\prime}$ is topologically finitely generated, and suppose, moreover, that $V$ is finite-dimensional. Then the same arguments as in [ST3], before Lemma 6.1, show that (2.4.1) is an isomorphism of topological vector spaces, if we give the left side the quotient topology of the projective tensor product topology on $D(H, K) \otimes_{K} V^{\prime} 3$ We will only consider induced representations where these conditions are met. (For more general situations cf. [K2, Proposition 5.3 and Remark 5.4.)

\footnotetext{
${ }^{3}$ We remark that the projective and inductive tensor product topologies coincide for tensor products of Fréchet spaces, cf. [S], Proposition 17.6.
} 


\section{Representations indUCED FROM A PARABOLIC SUBGROUP}

\subsection{The setting and statement of the main result.}

3.1.1. We consider a connected reductive algebraic group $\mathfrak{G}$ over the finite extension $L$ of $\mathbb{Q}_{p}$. Let $\mathfrak{S} \subset \mathfrak{G}$ be a maximal split torus over $L$. Fix a minimal parabolic subgroup $\mathfrak{P}_{0}$ of $\mathfrak{G}$ which contains $\mathfrak{S}$ and denote by $\mathfrak{U}_{0}$ its unipotent radical. The choice of $\mathfrak{P}_{0}$ determines subsets $\Phi^{+} \supset \Delta$ of positive and simple roots, respectively, in the root system $\Phi$ of $\mathfrak{G}$ with respect to $\mathfrak{S}$. Let $\mathfrak{P}$ be a parabolic subgroup containing $\mathfrak{P}_{0}$ with unipotent radical $\mathfrak{U}$. Let $\mathfrak{M}$ be the Levi subgroup of $\mathfrak{P}$ containing $\mathfrak{S}$. Let $W$ be the Weyl group of $\mathfrak{G}$ with respect to $\mathfrak{S}$, and let $W_{\mathbf{P}} \subset W$ be the Weyl group of the Levi subgroup $\mathfrak{M}$. Finally, we denote by $\Phi^{\text {red }}$ the set of reduced roots of $\Phi$.

We denote the corresponding groups of $L$-valued points by bold letters:

$$
\mathbf{G}=\mathfrak{G}(L), \mathbf{P}=\mathfrak{P}(L), \mathbf{S}=\mathfrak{S}(L), \text { etc., }
$$

which we consider as locally $L$-analytic groups. The Lie algebras will be denoted by gothic letters, i.e.,

$$
\mathfrak{g}=\operatorname{Lie}(\mathbf{G}), \mathfrak{p}=\operatorname{Lie}(\mathbf{P}), \mathfrak{s}=\operatorname{Lie}(\mathbf{S}), \text { etc. }
$$

\subsubsection{We let}

$$
\rho: \mathbf{P} \longrightarrow \mathbf{M} \longrightarrow \mathrm{GL}(V)
$$

be a representation of $\mathbf{P}$ which comes by inflation from a locally $L$-analytic representation of $\mathbf{M}$ on a finite-dimensional $K$-vector space $V$. We are interested in the locally analytic induced representation

$$
\operatorname{Ind}_{\mathbf{P}}^{\mathbf{G}}(\rho)
$$

and our main result gives a criterion for the topological irreducibility of this locally $L$-analytic representation of $\mathbf{G}$ in terms of the generalized Verma module

$$
m(\rho):=U(\mathfrak{g}) \otimes_{U(\mathfrak{p})} \rho^{\prime} .
$$

Here $\rho^{\prime}$ is the derived representation of $\mathfrak{p}$ on the dual space $V^{\prime}=\operatorname{Hom}_{K}(V, K)$. Our main result then is

Theorem 3.1.3. If $m(\rho)$ is a simple $U(\mathfrak{g})$-module, then $\operatorname{Ind}_{\mathbf{P}}^{\mathbf{G}}(\rho)$ is a topologically irreducible representation.

\subsection{The structure of the proof.}

3.2.1. Reduction to groups of the type (torus) $\times$ (simply connected). The first step is to reduce to the case where $\mathfrak{G}$ is a product of a torus and a semi-simple simply connected algebraic group 4 To this end let $\tilde{\mathfrak{G}}_{\text {der }}$ be the simply connected cover of the derived group $\mathfrak{G}_{\text {der }}$ of $\mathfrak{G}$. Let $\mathfrak{Z}$ be the connected component of the center of $\mathfrak{G}$. Put $\tilde{\mathfrak{G}}=\mathfrak{Z} \times \tilde{\mathfrak{G}}_{\text {der }}$. There is a canonical morphism $\iota: \tilde{\mathfrak{G}} \rightarrow \mathfrak{G}$ which induces an isomorphism between the corresponding Lie algebras. Put $\tilde{\mathfrak{P}}=\iota^{-1}(\mathfrak{P})$, which is a parabolic subgroup of $\tilde{\mathfrak{G}}$. The morphism $\iota$ indices an isomorphism of flag varieties $\tilde{\mathfrak{G}} / \tilde{\mathfrak{P}} \stackrel{\simeq}{\longrightarrow} \mathfrak{G} / \mathfrak{P}$, from which we deduce an isomorphism of representations of $\tilde{\mathbf{G}}$ :

$$
\operatorname{Ind}_{\mathbf{P}}^{\mathbf{G}}(\rho) \stackrel{\simeq}{\longrightarrow} \operatorname{Ind}_{\tilde{\mathbf{P}}}^{\tilde{\mathbf{G}}}(\tilde{\rho}) \text {. }
$$

\footnotetext{
${ }^{4}$ This is needed to ensure that the Iwahori subgroup $I$, to be defined below, possesses Iwahori decompositions w.r.t. all parabolic subgroups $w \mathbf{P} w^{-1}$, cf. 3.3 .1
} 
Here $\tilde{\rho}$ is the representation of $\tilde{\mathbf{P}}$ given by the composition of the induced map $\tilde{\mathbf{P}} \rightarrow \mathbf{P}$ and $\rho$. The action of $\tilde{\mathbf{G}}$ on the right comes from the homomorphism $\tilde{\mathbf{G}} \rightarrow \mathbf{G}$. It follows that $\operatorname{Ind}_{\mathbf{P}}^{\mathbf{G}}(\rho)$ is a (topologically) irreducible $\mathbf{G}$-representation if $\operatorname{Ind}_{\tilde{\mathbf{P}}}^{\tilde{\mathbf{G}}}(\tilde{\rho})$ is a (topologically) irreducible $\tilde{\mathbf{G}}$-representation. The same argument applies to the Verma modules. Therefore, we will assume from now on that $\mathfrak{G}$ is the product of a central torus and a semi-simple and simply connected group.

3.2.2. Passage to representations of compact groups. The second step is to reduce the analysis of the induced representation to a question about representations of compact groups. To this end we fix a special maximal compact subgroup $G \subset \mathbf{G}$. From the Iwasawa decomposition $\mathbf{G}=G \cdot \mathbf{P}$ (cf. Ca], sec. 3.5), we deduce an isomorphism of $G$-representations

$$
\left.\operatorname{Ind}_{\mathbf{P}}^{\mathbf{G}}(\rho)\right|_{G} \stackrel{\simeq}{\longrightarrow} \operatorname{Ind}_{G \cap \mathbf{P}}^{G}(\rho) .
$$

We let $I \subset G$ be an Iwahori subgroup, and put $P^{+}=G \cap \mathbf{P}$. We note that every element of the Weyl group $W$ of $\mathfrak{G}$ with respect to $\mathfrak{S}$ has a representative in $G$ (cf. BT1, 4.2.3, Ca, p. $140(\mathrm{~b})$ ), and hence we identify $W$ with $\left(N_{\mathbf{G}}(\mathbf{S}) \cap\right.$ $G) /\left(Z_{\mathbf{G}}(\mathbf{S}) \cap G\right)$. The same remark applies to the Weyl group $W_{\mathbf{P}}$. From the Bruhat-Tits decomposition (cf. [Ca], 3.5) we deduce that

$$
\mathbf{G}=\coprod_{w \in W / W_{\mathbf{P}}} I w \mathbf{P} .
$$

It follows that

$$
G=\coprod_{w \in W / W_{\mathbf{P}}} I w P^{+} .
$$

This in turn shows that, as representations of $I$, we have

$$
\left.\operatorname{Ind}_{G \cap \mathbf{P}}^{G}(\rho)\right|_{I} \cong \bigoplus_{w \in W / W_{\mathbf{P}}} \operatorname{Ind}_{P_{w}^{+}}^{I}\left(\rho^{w}\right),
$$

where $P_{w}^{+}=I \cap w P^{+} w^{-1}$. Here, we fix once and for all representatives for elements in $W / W_{\mathbf{P}}$ as follows: first we take from each coset $w W_{\mathbf{P}}$ its Kostant representatives, $\dot{w}$ say, and then we fix a representative of $\dot{w}$ in $G$ (that is possible, cf. above). When we write $w \in W / W_{\mathbf{P}}$, then $w$ denotes this specifically chosen representative in $G$. We let $M^{w}(\rho)$ be the $D(P, K)$-module dual to $\operatorname{Ind}_{P_{w}^{+}}^{I}\left(\rho^{w}\right)$, i.e.,

$$
M^{w}(\rho)=\left(\operatorname{Ind}_{P_{w}^{+}}^{I}\left(\rho^{w}\right)\right)_{b}^{\prime}=D(I, K) \otimes_{D\left(P_{w}^{+}, K\right)} V_{w}^{\prime},
$$

where $V_{w}$ denotes the vector space $V$ equipped with the action $\rho^{w}$ of $P_{w}^{+}$given by $\rho^{w}(h)=\rho\left(w^{-1} h w\right)$, cf. 2.4.1). We get

$$
\left(\operatorname{Ind}_{G \cap \mathbf{P}}^{G}(\rho)\right)_{b}^{\prime}=\bigoplus_{w \in W / W_{\mathbf{P}}} M^{w}(\rho) .
$$

Assuming that all $D(I, K)$-modules $M^{w}(\rho)$ are simple we show in Proposition 3.5.1 that they are pairwise non-isomorphic. This implies that the left-hand side is a simple $D(G, K)$-module, which in turn shows that $\operatorname{Ind}_{G \cap \mathbf{P}}^{G}(\rho)$ is a topologically irreducible $G$-representation. 
3.2.3. From $D(I, K)$-modules to $D_{r}(I, K)$-modules. Because the representation $V$ is finite-dimensional, the canonical map $V_{w}^{\prime} \rightarrow D_{r}\left(P_{w}^{+}, K\right) \otimes_{D\left(P_{w}^{+}, K\right)} V_{w}^{\prime}$ is an isomorphism for $r<1$ sufficiently close to 1 , cf. Proposition 3.4.2. Hence

$$
\begin{aligned}
M_{r}^{w}(\rho) & :=D_{r}(I, K) \otimes_{D(I, K)} M^{w}(\rho)=D_{r}(I, K) \otimes_{D\left(P_{w}^{+}, K\right)} V_{w}^{\prime} \\
& =D_{r}(I, K) \otimes_{D_{r}\left(P_{w}^{+}, K\right)}\left(D_{r}\left(P_{w}^{+}, K\right) \otimes_{D\left(P_{w}^{+}, K\right)} V_{w}^{\prime}\right) \\
& =D_{r}(I, K) \otimes_{D_{r}\left(P_{w}^{+}, K\right)} V_{w}^{\prime} .
\end{aligned}
$$

By [ST2, Lemma 3.9, $M^{w}(\rho)$ is a simple $D(I, K)$-module if $M_{r}^{w}(\rho)$ is a simple $D_{r}(I, K)$-module for a sequence of $r$ 's tending to 1 , so we are done if we show that all $M_{r}^{w}(\rho)$ are simple $D_{r}(I, K)$-modules, assuming the simplicity of $m(\rho)$ as an $U(\mathfrak{g})$-module.

In order to study the modules $M_{r}^{w}(\rho)$ we use the Iwahori product decomposition, cf. Lemma 3.3.2

$$
I=U_{w}^{-} \cdot P_{w}^{+},
$$

where $U_{w}^{-}=I \cap w \mathbf{U}^{-} w^{-1}$ and $\mathbf{U}^{-}$is the unipotent radical of the parabolic subgroup opposite to $\mathbf{P}$. We choose an open uniform normal subgroup $I_{0} \subset I$ such that

$$
I_{0}=U_{w, 0}^{-} \cdot P_{w, 0}^{+} \text {where } U_{w, 0}^{-}=U_{w}^{-} \cap I_{0} \text { and } P_{w, 0}^{+}=P_{w}^{+} \cap I_{0} .
$$

We will show that it is possible to choose $I_{0}$ in such a way that $P_{w, 0}^{+}$is uniform and $U_{w, 0}^{-}$is $L$-uniform (cf. Remark 2.2.5). The completed distribution algebras of $I\left(U_{w}^{-}\right.$and $P_{w}^{+}$, resp.) are defined by means of the canonical $p$-valuation on $I_{0}\left(U_{w, 0}^{-}\right.$ and $P_{w, 0}^{+}$, resp.), cf. 2.2.6. For $r<1$ sufficiently close to 1 , we have a canonical isomorphism of Banach spaces

$$
D_{r}\left(U_{w}^{-}, K\right) \hat{\otimes}_{K} D_{r}\left(P_{w}^{+}, K\right) \stackrel{\simeq}{\longrightarrow} D_{r}(I, K),
$$

which in turn gives rise to a canonical isomorphism of $D_{r}\left(U_{w}^{-}, K\right)$-modules

$$
D_{r}\left(U_{w}^{-}, K\right) \otimes_{K} V_{w}^{\prime} \stackrel{\simeq}{\longrightarrow} M_{r}^{w}(\rho),
$$

cf. Proposition 3.4.2. Using the integrality of the distribution algebra $D_{r}\left(U_{w}^{-}, K\right)$, cf. Proposition 3.3.5 together with Corollary 2.3.4 we prove that any non-zero $D_{r}(I, K)$-submodule $N$ of $M_{r}^{w}(\rho)$ has non-zero intersection with

$$
m_{r}^{w}(\rho):=U_{r}\left(\mathfrak{u}_{w}^{-}, U_{w, 0}^{-}\right) \otimes_{K} V_{w}^{\prime},
$$

cf. Proposition 3.4.10. Using general results about orthogonal bases we can even infer that $N$ has non-zero intersection with

$$
m^{w}(\rho)=U\left(\mathfrak{u}_{w}^{-}\right) \otimes_{K} V_{w}^{\prime} \simeq U(\mathfrak{g}) \otimes_{U\left(\mathfrak{p}_{w}^{+}\right)} V_{w}^{\prime} .
$$

But it is not difficult to see that if $m(\rho):=m^{1}(\rho)=U(\mathfrak{g}) \otimes_{\mathfrak{p}} V^{\prime}$ is a simple $U(\mathfrak{g})$-module, then $m^{w}(\rho)$ is a simple $U(\mathfrak{g})$-module for all $w$, and this implies that $M_{r}^{w}(\rho)$ is a simple $D_{r}(I, K)$-module for all $w$ and all $r$ sufficiently close to 1 . Hence, by our previous remark, $M^{w}(\rho)$ is a simple $D(I, K)$-module for all $w$. From what we have said at the end of 3.2 .2 it then follows that $\operatorname{Ind}_{G \cap \mathbf{P}}^{G}(\rho)$ is a topologically irreducible $G$-representation. 


\subsection{Parahoric subgroups and their distribution algebras.}

3.3.1. For the following compare $\mathrm{Ca}$, sec. 3.5. The torus $\mathfrak{S}$ determines an apartment $\mathcal{A}$ in the Bruhat-Tits building of $\mathfrak{G}$ over $L$. We fix a special vertex $x_{0}$ in the apartment $\mathcal{A}$. Then there is a unique conical chamber $\mathcal{C}$ in $\mathcal{A}$ having $x_{0}$ as apex and satisfying the following property: for every $u$ in the unipotent radical $\mathbf{U}_{0}$ of $\mathbf{P}_{0}$ the intersection $\mathcal{C} \cap u \mathcal{C}$ contains a translate of $\mathcal{C}$. Moreover, there is a unique chamber $C_{0}$ in $\mathcal{C}$ having $x_{0}$ as one of its vertices. We let $G \subset \mathbf{G}$ be the stabilizer of $x_{0}$ and $I \subset G$ the pointwise stabilizer of $C_{0}$. Let $\mathbf{U}^{-}$be the unipotent radical of the parabolic subgroup of $\mathbf{G}$ opposite to $\mathbf{P}$. For $w \in W / W_{\mathbf{P}}$, we put

$$
P_{w}^{+}=I \cap w \mathbf{P} w^{-1}, U_{w}^{+}=I \cap w \mathbf{U} w^{-1}, U_{w}^{-}=I \cap w \mathbf{U}^{-} w^{-1} .
$$

Lemma 3.3.2. The multiplication map

$$
P_{w}^{+} \times U_{w}^{-} \longrightarrow I
$$

is an isomorphism of locally L-analytic manifolds. In particular, there are decompositions

$$
I=P_{w}^{+} \cdot U_{w}^{-}=U_{w}^{-} \cdot P_{w}^{+} .
$$

Proof. Let $\mathbf{Z}$ be the centralizer of $\mathbf{S}$ in $\mathbf{G}$ and denote by $Z_{c}$ the kernel of the natural homomorphism $\mathbf{Z}(L) \rightarrow X_{*}(S)_{\mathbb{R}}$. For a (reduced) root $\alpha \in \Phi^{\text {red }}$, let $\mathbf{U}_{\alpha}$ be its root subgroup in $\mathbf{G}$. Let $X_{\alpha}=\mathbf{U}_{\alpha}(L) \cap I$. By our assumption on $\mathfrak{G}$, the condition in Ti], the first sentence in 3.1.1, is met (cf. the sentence preceding sec. 3.1.1 in [Ti]). Therefore, by the last sentence in [Ti], sec. 3.1.1, we have a product decomposition

$$
I=Z_{c} \cdot \prod_{\alpha \in \Phi^{\mathrm{red}}} X_{\alpha}
$$

where the product is taken with respect to any ordering on the roots. The claim follows immediately.

3.3.3. Defining norms on $D(I, K)$. We start with the construction of the subgroup $I_{0} \subset I$ mentioned in 3.2 .3 . It follows from $[\mathrm{S}-\mathrm{S}]$, Proposition I.2.7, that the subgroups $U_{x_{0}}^{(e)}$ of $G$ constructed there (we take for the facet $F$ in $[\mathrm{S}-\mathrm{S}]$ the special vertex $x_{0}$ of 3.3.1) possess an Iwahori decomposition with respect to the parabolic subgroup $\mathbf{P}$ :

$$
U_{x_{0}}^{(e)}=\left(U_{x_{0}}^{(e)} \cap \mathbf{U}^{-}\right) \cdot\left(U_{x_{0}}^{(e)} \cap \mathbf{P}\right) .
$$

But as the groups $U_{x_{0}}^{(e)}$ are normal in $G(\underline{\underline{S}-\mathrm{S}}$, three lines before Proposition I.2.7), they possess an Iwahori decomposition with respect to any parabolic subgroup of the form $w \mathbf{P} w^{-1}$ :

$$
U_{x_{0}}^{(e)}=w\left(U_{x_{0}}^{(e)} \cap \mathbf{U}^{-}\right) w^{-1} \cdot w\left(U_{x_{0}}^{(e)} \cap \mathbf{P}\right) w^{-1}=\left(U_{x_{0}}^{(e)} \cap w \mathbf{U}^{-} w^{-1}\right) \cdot\left(U_{x_{0}}^{(e)} \cap w \mathbf{P} w^{-1}\right) .
$$

The groups $U_{x_{0}}^{(e)}$ form a fundamental system of compact open neighborhoods of 1 in $\mathbf{G}$ ([S-S], Cor. I.2.9). Fix an $L$-uniform subgroup $H \subset I$ (cf. Remark 2.2.5). Then there is $e>0$ such that $U_{x_{0}}^{(e)} \subset H$. Let $\Lambda \subset \operatorname{Lie}(H)=\mathfrak{g}$ be the $\mathfrak{o}_{L}$ lattice from 
Lemma 2.2.4 i.e., $\exp _{H}$ maps $\Lambda$ homeomorphically onto $H$. Define:

$$
\begin{aligned}
& \Lambda_{0}:=\exp _{H}^{-1}\left(U_{x_{0}}^{(e)}\right), \\
& \Lambda_{1}:=\exp _{H}^{-1}\left(U_{x_{0}}^{(e)} \cap w \mathbf{U}^{-} w^{-1}\right), \\
& \Lambda_{2}:=\exp _{H}^{-1}\left(U_{x_{0}}^{(e)} \cap w \mathbf{P} w^{-1}\right) .
\end{aligned}
$$

Each $\Lambda_{i}, i=0,1,2$, is a $\mathbb{Z}_{p}$-lattice in $\Lambda$. For $t \gg 0$ we then have that $p^{t} \Lambda_{i}$ is a uniform $\mathbb{Z}_{p}$-Lie algebra in the sense of [DDMS, sec. 9.4. Fix such a $t$ and define

$$
\begin{aligned}
& U_{w, 0}^{-}:=\exp _{H}\left(p^{t} \Lambda_{1}\right) \subset U_{x_{0}}^{(e)} \cap w \mathbf{U}^{-} w^{-1} \subset U_{w}^{-}, \\
& P_{w, 0}^{+}:=\exp _{H}\left(p^{t} \Lambda_{2}\right) \subset U_{x_{0}}^{(e)} \cap w \mathbf{P} w^{-1} \subset P_{w}^{+}, \\
& I_{0} \quad:=\exp _{H}\left(p^{t} \Lambda_{0}\right) \subset U_{x_{0}}^{(e)} \quad \subset \quad I .
\end{aligned}
$$

The subgroup $U_{w, 0}^{-}\left(P_{w, 0}^{+}, I_{0}\right.$, resp. $)$ consists of the $p^{t}$-th powers of elements of $U_{x_{0}}^{(e)} \cap w \mathbf{U}^{-} w^{-1}\left(U_{x_{0}}^{(e)} \cap w \mathbf{P} w^{-1}, U_{x_{0}}^{(e)}\right.$, resp.), and is thus a characteristic subgroup of $U_{x_{0}}^{(e)} \cap w \mathbf{U}^{-} w^{-1}\left(U_{x_{0}}^{(e)} \cap w \mathbf{P} w^{-1}, U_{x_{0}}^{(e)}\right.$, resp.). As $U_{x_{0}}^{(e)}$ is normal in $G$, it follows that the group $U_{x_{0}}^{(e)} \cap w \mathbf{U}^{-} w^{-1}\left(U_{x_{0}}^{(e)} \cap w \mathbf{P} w^{-1}, U_{x_{0}}^{(e)}\right.$, resp.) is normal in $U_{w}^{-}\left(P_{w}^{+}, I\right.$, resp.). And this implies that $U_{w, 0}^{-}\left(P_{w, 0}^{+}, I_{0}\right.$, resp.) is normal in $U_{w}^{-}\left(P_{w}^{+}, I\right.$, resp.). Each group $U_{w, 0}^{-}, P_{w, 0}^{+}$, and $I_{0}$, is uniform pro- $p$.

We now show that $U_{w, 0}^{-}$is actually $L$-uniform. Because of the root decomposition of $U_{x_{0}}^{(e)}$ in $\underline{[S-S}$, Proposition I.2.7, it suffices to consider $U_{w, 0}^{-} \cap \mathbf{U}_{\alpha}$ for some $\alpha \in\left(\Phi^{-}\right.$) $\left.\Phi_{\mathbf{P}}\right) \cap \Phi^{\text {red }}$. Here, $\Phi_{\mathbf{P}}$ is the root system of the Levi subgroup of $\mathfrak{P}$ which contains $\mathfrak{S}$, and $\mathbf{U}_{\alpha}$ is the generalized root group with Lie algebra $\mathfrak{g}_{\alpha} \oplus \mathfrak{g}_{2 \alpha} 5$ It follows from BT2, the last sentence in sec. 5.2.2, that $U_{x_{0}}^{(e)} \cap \mathbf{U}_{\alpha}$ is equal to $\mathcal{U}\left(\mathfrak{o}_{L}\right)$, where $\mathcal{U}$ is a group scheme over $\mathfrak{o}_{L}$ which is, as a scheme, isomorphic to $\operatorname{Spec}\left(\operatorname{Sym}_{\mathfrak{o}_{L}}(N)\right)$ with a free $\mathfrak{o}_{L}$-module $N$ of finite rank. Hence, we see that $U_{w, 0}^{-} \cap \mathbf{U}_{\alpha}=\mathcal{U}_{t}\left(\mathfrak{o}_{L}\right)$, where $\mathcal{U}_{t}=\operatorname{Spec}\left(\operatorname{Sym}_{\mathfrak{o}_{L}}\left(N_{t}\right)\right)$ with $N_{t}=p^{-t} N$. The group of $\mathfrak{o}_{L}$-valued points $\mathcal{U}_{t}\left(\mathfrak{o}_{L}\right)$ is identified with the $\mathfrak{o}_{L}$-algebra homomorphisms $\operatorname{Sym}_{\mathfrak{o}_{L}}\left(N_{t}\right) \rightarrow \mathfrak{o}_{L}$, which are uniquely determined by restriction to $N_{t} \subset \operatorname{Sym}_{\mathfrak{o}_{L}}\left(N_{t}\right)$. We may assume that the zero section of $\mathcal{U}_{t}$ corresponds to the map which sends $N_{t}$ to zero. Let $\left(N_{t}\right) \subset$ $\operatorname{Sym}_{\mathfrak{o}_{L}}\left(N_{t}\right)$ be the ideal generated by $N_{t}$. Then the Lie algebra of the group scheme $\mathcal{U}_{t}$ can be identified with the relative tangent space $\operatorname{Hom}_{\mathfrak{o}_{L}}\left(\left(N_{t}\right) /\left(N_{t}\right)^{2}, \mathfrak{o}_{L}\right)$. It is easy to see that the exponential map

$$
\exp _{\mathcal{U}_{t}\left(\mathfrak{o}_{L}\right)}: \operatorname{Lie}\left(\mathcal{U}_{t}\right) \otimes_{\mathfrak{o}_{L}} L \rightarrow \mathcal{U}_{t}\left(\mathfrak{o}_{L}\right),
$$

when restricted to a sufficiently small submodule of $\operatorname{Lie}\left(\mathcal{U}_{t}\right)$, corresponds then to the restriction (to that submodule) of the composed map

$$
\begin{aligned}
\operatorname{Lie}\left(\mathcal{U}_{t}\right)=\operatorname{Hom}_{\mathfrak{o}_{L}}\left(\left(N_{t}\right) /\left(N_{t}\right)^{2}, \mathfrak{o}_{L}\right) & \longrightarrow \operatorname{Hom}_{\mathfrak{o}_{L}}\left(N_{t}, \mathfrak{o}_{L}\right) \\
& \longrightarrow \operatorname{Hom}_{\mathfrak{o}_{L} \text {-algebras }}\left(\operatorname{Sym}_{\mathfrak{o}_{L}}\left(N_{t}\right), \mathfrak{o}_{L}\right)=\mathcal{U}_{t}\left(\mathfrak{o}_{L}\right) .
\end{aligned}
$$

\footnotetext{
${ }^{5}$ Here $\mathfrak{g}_{2 \alpha}=0$ if $2 \alpha$ is not a root.
} 
The first map is defined by restricting a map $\left(N_{t}\right) /\left(N_{t}\right)^{2} \rightarrow \mathfrak{o}_{L}$ to $N_{t} \subset\left(N_{t}\right) /\left(N_{t}\right)^{2}$. The maps in this sequence are all bijections. Because $\mathcal{U}_{t}\left(\mathfrak{o}_{L}\right)$ is uniform pro- $p$, the exponential map maps a lattice in $\operatorname{Lie}\left(\mathcal{U}_{t}\right)$ bijectively onto $\mathcal{U}_{t}\left(\mathfrak{o}_{L}\right)$, and this lattice must therefore be $\operatorname{Lie}\left(\mathcal{U}_{t}\right)$, which is an $\mathfrak{o}_{L}$-module. Hence, we conclude that $U_{w, 0}^{-} \cap \mathbf{U}_{\alpha}=\mathcal{U}_{t}\left(\mathfrak{o}_{L}\right)$ is $L$-uniform, and it follows that $U_{w, 0}^{-}$is $L$-uniform as well.

We have $I_{0}=U_{w, 0}^{-} \cdot P_{w, 0}^{+}$, and for the canonical $p$-valuation on $I_{0}$ it follows from the identity $P_{i+1}\left(I_{0}\right)=\exp \left(p^{i} \cdot p^{t} \Lambda_{0}\right)$, together with DDMS], Lemma 4.14 (iv), that for $x \in p^{t} \Lambda_{1}$ and $y \in p^{t} \Lambda_{2}$ we have

$$
\omega^{\text {can }}(\exp (x) \exp (y))=\varepsilon_{p}+\min \{a, b\}=\min \left\{\omega^{c a n}(\exp (x)), \omega^{c a n}(\exp (y))\right\},
$$

where $a, b$ are such that $x \in p^{a} \cdot\left(p^{t} \Lambda_{1}\right) \backslash p^{a+1} \cdot\left(p^{t} \Lambda_{1}\right)$ and $y \in p^{b} \cdot\left(p^{t} \Lambda_{2}\right) \backslash p^{b+1} \cdot\left(p^{t} \Lambda_{2}\right)$ (with $a$ (resp. $b$ ) being $\infty$ if $x=0$ (resp. $y=0)$ ). In order to define the norms $q_{r}$ on the rings $D\left(U_{w}^{-}, K\right), D\left(P_{w}^{+}, K\right)$ and $D(I, K)$, we work with the uniform normal subgroups $U_{w, 0}^{-}, P_{w, 0}^{+}$and $I_{0}$, and the canonical $p$-valuations on these groups, following the recipe explained in 2.2.6.

In the following proposition, all completed tensor products are meant to be the completions of the ordinary tensor products with respect to the projective tensor product topology, cf. [S], sec. 17. We remark that for Fréchet spaces the projective and the inductive tensor product topology coincide, cf. [S], Proposition 17.6.

Proposition 3.3.4. (i) The decompositions $I=U_{w}^{-} \cdot P_{w}^{+}, I_{0}=U_{w, 0}^{-} \cdot P_{w, 0}^{+}$induce isomorphisms of topological $K$-vector spaces

$$
\begin{aligned}
C_{\mathbb{Q}_{p}}^{a n}\left(U_{w}^{-}, K\right) \hat{\otimes}_{K} C_{\mathbb{Q}_{p}}^{a n}\left(P_{w}^{+}, K\right) \stackrel{\simeq}{\longrightarrow} C_{\mathbb{Q}_{p}}^{a n}(I, K), \\
C_{\mathbb{Q}_{p}}^{a n}\left(U_{w, 0}^{-}, K\right) \hat{\otimes}_{K} C_{\mathbb{Q}_{p}}^{a n}\left(P_{w, 0}^{+}, K\right) \stackrel{\simeq}{\longrightarrow} C_{\mathbb{Q}_{p}}^{a n}\left(I_{0}, K\right), \\
C_{L}^{a n}\left(U_{w}^{-}, K\right) \hat{\otimes}_{K} C_{L}^{a n}\left(P_{w}^{+}, K\right) \stackrel{\simeq}{\longrightarrow} C_{L}^{a n}(I, K), \\
C_{L}^{a n}\left(U_{w, 0}^{-}, K\right) \hat{\otimes}_{K} C_{L}^{a n}\left(P_{w, 0}^{+}, K\right) \stackrel{\simeq}{\longrightarrow} C_{L}^{a n}\left(I_{0}, K\right) .
\end{aligned}
$$

(ii) We equip the rings $D_{\mathbb{Q}_{p}}\left(I_{0}, K\right), D_{\mathbb{Q}_{p}}\left(U_{w, 0}^{-}, K\right)$ and $D_{\mathbb{Q}_{p}}\left(P_{w, 0}^{+}, K\right)$ with the norm $\|\cdot\|_{r}, \frac{1}{p}<r<1$, associated to the canonical p-valuation. The rings $D_{\mathbb{Q}_{p}}(I, K)$, $D_{\mathbb{Q}_{p}}\left(U_{w}^{-}, K\right)$ and $D_{\mathbb{Q}_{p}}\left(P_{w}^{+}, K\right)$ carry the maximum norms $q_{r}$, and $D_{L}(I, K), \quad D_{L}\left(U_{w}^{-}, K\right), \quad D_{L}\left(P_{w}^{+}, K\right)$ as well as $D_{L}\left(I_{0}, K\right), D_{L}\left(U_{w, 0}^{-}, K\right)$ and $D_{L}\left(P_{w, 0}^{+}, K\right)$ are equipped with the quotient norms $\bar{q}_{r}$. On the tensor products of these spaces we put the usual induced norm. Then the isomorphisms in (i) induce isometries of topological $K$-vector spaces

$$
\begin{aligned}
D_{\mathbb{Q}_{p}}(I, K) \stackrel{\simeq}{\longrightarrow} D_{\mathbb{Q}_{p}}\left(U_{w}^{-}, K\right) \hat{\otimes}_{K} D_{\mathbb{Q}_{p}}\left(P_{w}^{+}, K\right), \\
D_{\mathbb{Q}_{p}}\left(I_{0}, K\right) \stackrel{\simeq}{\longrightarrow} D_{\mathbb{Q}_{p}}\left(U_{w, 0}^{-}, K\right) \hat{\otimes}_{K} D_{\mathbb{Q}_{p}}\left(P_{w, 0}^{+}, K\right), \\
D_{L}(I, K) \stackrel{\simeq}{\longrightarrow} D_{L}\left(U_{w}^{-}, K\right) \hat{\otimes}_{K} D_{L}\left(P_{w}^{+}, K\right), \\
D_{L}\left(I_{0}, K\right) \stackrel{\simeq}{\longrightarrow} D_{L}\left(U_{w, 0}^{-}, K\right) \hat{\otimes}_{K} D_{L}\left(P_{w, 0}^{+}, K\right) .
\end{aligned}
$$


(iii) The isometries in (ii) furnish isometries of the completions

$$
\begin{gathered}
D_{r}(I, K) \stackrel{\simeq}{\longrightarrow} D_{r}\left(U_{w}^{-}, K\right) \hat{\otimes}_{K} D_{r}\left(P_{w}^{+}, K\right), \\
D_{r}\left(I_{0}, K\right) \stackrel{\simeq}{\longrightarrow} D_{r}\left(U_{w, 0}^{-}, K\right) \hat{\otimes}_{K} D_{r}\left(P_{w, 0}^{+}, K\right) .
\end{gathered}
$$

Proof. (i) This follows from [ST3], Lemma A.1 and Proposition A.2.

(ii) Let $h_{1}, \ldots, h_{d^{\prime}}$ be a $p$-basis of $U_{w, 0}^{-}$and $h_{d^{\prime}+1}, \ldots, h_{d}$ a $p$-basis of $P_{w, 0}^{+}$. For $u \in U_{w, 0}^{-}, p \in P_{w, 0}^{+}$, we have by the discussion in 3.3 .3

$$
\omega^{c a n}(u p)=\min \left\{\omega^{c a n}(u), \omega^{c a n}(p)\right\},
$$

so that $h_{1}, \ldots, h_{d^{\prime}}, h_{d^{\prime}+1}, \ldots, h_{d}$ is a $p$-basis of $I_{0}$. Elements of $D_{\mathbb{Q}_{p}}\left(I_{0}, K\right)$ have a unique expansion as a series of the form

$$
\sum_{n \in \mathbb{N}_{0}^{d}} d_{n} \mathbf{b}^{n}
$$

with $\mathbf{b}^{n}=\left(h_{1}-1\right)^{n_{1}} \cdot \ldots \cdot\left(h_{d}-1\right)^{n_{d}}$. From this we deduce immediately that the canonical map

$$
D_{\mathbb{Q}_{p}}\left(I_{0}, K\right) \stackrel{\simeq}{\longrightarrow} D_{\mathbb{Q}_{p}}\left(U_{w, 0}^{-}, K\right) \hat{\otimes}_{K} D_{\mathbb{Q}_{p}}\left(P_{w, 0}^{+}, K\right)
$$

is an isometry when equipped with the norm $\|\cdot\|_{r}$ on the left-hand side and with the induced norm on the tensor product. Consider the canonical commutative diagram

$$
\begin{array}{cccc}
D_{\mathbb{Q}_{p}}\left(I_{0}, K\right) & \stackrel{\simeq}{\longrightarrow} & D_{\mathbb{Q}_{p}}\left(U_{w, 0}^{-}, K\right) \hat{\otimes}_{K} D_{\mathbb{Q}_{p}}\left(P_{w, 0}^{+}, K\right) \\
\downarrow & & \downarrow \\
D_{L}\left(I_{0}, K\right) & \stackrel{\simeq}{\longrightarrow} & D_{L}\left(U_{w, 0}^{-}, K\right) \hat{\otimes}_{K} D_{L}\left(P_{w, 0}^{+}, K\right)
\end{array}
$$

Let $\bar{q}_{r}$ denote the quotient norm on $D_{L}\left(U_{w, 0}^{-}, K\right)$ as well as on $D_{L}\left(P_{w, 0}^{+}, K\right)$, and let $\bar{q}_{r} \otimes \bar{q}_{r}$ be the induced norm on the tensor product. On the other hand, let $\|\cdot\|_{r} \otimes\|\cdot\|_{r}$ be the norm on $D_{\mathbb{Q}_{p}}\left(U_{w, 0}^{-}, K\right) \hat{\otimes}_{K} D_{\mathbb{Q}_{p}}\left(P_{w, 0}^{+}, K\right)$, and denote by $\overline{\|\cdot\|_{r} \otimes\|\cdot\|_{r}}$ the norm on $D_{L}\left(U_{w, 0}^{-}, K\right) \hat{\otimes}_{K} D_{L}\left(P_{w, 0}^{+}, K\right)$ induced by the surjection

$$
D_{\mathbb{Q}_{p}}\left(U_{w, 0}^{-}, K\right) \hat{\otimes}_{K} D_{\mathbb{Q}_{p}}\left(P_{w, 0}^{+}, K\right) \rightarrow D_{L}\left(U_{w, 0}^{-}, K\right) \hat{\otimes}_{K} D_{L}\left(P_{w, 0}^{+}, K\right) .
$$

By BGR, Ch. 2.1, Proposition 6, we know that

$$
\bar{q}_{r} \otimes \bar{q}_{r}=\overline{\|\cdot\|_{r} \otimes\|\cdot\|_{r}},
$$

which in turn shows that

$$
D_{L}\left(I_{0}, K\right) \stackrel{\simeq}{\longrightarrow} D_{L}\left(U_{w, 0}^{-}, K\right) \hat{\otimes}_{K} D_{L}\left(P_{w, 0}^{+}, K\right)
$$

is an isometry. Finally, using Remark 2.2.7 we can conclude that

$$
D_{L}(I, K) \stackrel{\simeq}{\longrightarrow} D_{L}\left(U_{w}^{-}, K\right) \hat{\otimes}_{K} D_{L}\left(P_{w}^{+}, K\right)
$$

is an isometry as well.

(iii) This statement follows from (ii).

Proposition 3.3.5. (i) Let $\mathfrak{H}$ be a connected reductive group over $L, \mathfrak{P} \subset \mathfrak{H}$ a parabolic subgroup (defined over $L$ ), and let $\mathfrak{N}$ be the unipotent radical of $\mathfrak{P}$. Consider a compact open subgroup $H \subset \mathfrak{N}(L)$. Choose an L-uniform subgroup $H_{0} \subset H$ (cf. Remark 2.2.5), and define the norm $\bar{q}_{r}$ on $D(H, K)$ as explained in 2.2.6 (using the canonical p-valuation on $\left.H_{0}\right)$. Then there is a sequence $\left(r_{m}\right)_{m \geq 1}$ of numbers in $\left(\frac{1}{p}, 1\right) \cap p^{\mathbb{Q}}$, and converging to 1 , such that the ring $D_{r_{m}}(H, K)$ is an integral domain for all $m$. 
(ii) There is a sequence $\left(r_{m}\right)_{m \geq 1}$ of numbers in $\left(\frac{1}{p}, 1\right) \cap p^{\mathbb{Q}}$, and converging to 1 , such that the ring $D_{r_{m}}\left(U_{w}^{-}, K\right)$ is an integral domain.

Proof. (i) Let $\bar{q}_{r}$ be the norm on $D_{r}(H, K)$ defined by means of the uniform subgroup $H_{0}$ (using the canonical $p$-valuation on $H_{0}$ ) as explained in 2.2.6. The key idea of the proof is to embed $D_{r}(H, K)$ in another distribution ring of the form $D_{r^{\prime}}\left(H^{\prime}, K\right)$ which is an integral domain. First we note that for any given compact open subgroup $H^{\prime} \subset \mathfrak{N}(L)$ there is an element $s$ in a (maximal) torus $\mathfrak{S}(L)$ (which is chosen to normalize $\mathfrak{N}(L)$ ) such that $s H^{-1}$ is contained in $H^{\prime}$. We choose a compact open subgroup $H^{\prime} \subset \mathfrak{N}(L)$ which is $L$-uniform (cf. Remark 2.2.5). $H$ and $s H s^{-1}$ being isomorphic locally $L$-analytic groups, it suffices to show the assertion for $s \mathrm{Hs}^{-1}$, i.e., we may assume without loss of generality that $H$ is already contained in a compact open subgroup $H^{\prime}$ which is $L$-uniform. The embedding $H \hookrightarrow H^{\prime}$ gives rise to a continuous embedding of Fréchet spaces

$$
D(H, K) \hookrightarrow D\left(H^{\prime}, K\right) .
$$

Denote by $\bar{q}_{r}^{H^{\prime}}$ the norms on $D\left(H^{\prime}, K\right)$ defined by means of the canonical $p$ valuation on $H^{\prime}$ ( $H^{\prime}$ is uniform pro- $p$ ). The topology of the Fréchet spaces $D(H, K)$ and $D\left(H^{\prime}, K\right)$, is defined by the family of norms $\left(\bar{q}_{r}\right)_{r}$ and $\left(\bar{q}_{r}^{H^{\prime}}\right)_{r}$, respectively. Therefore, for any $r^{\prime}$, there is an inequality of norms on $D(H, K)$,

$$
\bar{q}_{r^{\prime}}^{H^{\prime}} \leq c \bar{q}_{r}
$$

for $r$ sufficiently close to 1 . Next we consider the decomposition

$$
D\left(H^{\prime}, K\right)=\bigoplus_{h \in H^{\prime} / H} \delta_{h} \cdot D(H, K),
$$

and denote by $\mu_{r}$ the maximum norm on $D\left(H^{\prime}, K\right)$ induced by the norm $\bar{q}_{r}$ :

$$
\mu_{r}\left(\sum_{h \in H^{\prime} / H} \delta_{h} \cdot \lambda_{h}\right)=\max \left\{\bar{q}_{r}\left(\lambda_{h}\right) \mid h \in H^{\prime} / H\right\} .
$$

The norms $\mu_{r}$ may not be algebra norms in general, but they define the Fréchet topology on $D\left(H^{\prime}, K\right)$, as well. Hence for a given $r_{0}$, there is an inequality

$$
\mu_{r_{0}} \leq c^{\prime} \bar{q}_{r^{\prime}}^{H^{\prime}}
$$

for $r^{\prime}$ sufficiently close to 1 . Choose any $r_{0} \in\left(p^{-\frac{1}{\varepsilon_{p}(p-1)}}, 1\right)$. Then, by Proposition 5.2.1, there is $r^{\prime} \in\left(\frac{1}{p}, 1\right) \cap p^{\mathbb{Q}}$ such that (3.3.7) holds, and such that $D_{r^{\prime}}\left(H^{\prime}, K\right)$ is an integral domain. This shows that there is a continuous map

$$
D_{r^{\prime}}\left(H^{\prime}, K\right) \longrightarrow\left(D\left(H^{\prime}, K\right), \mu_{r_{0}}\right)^{\wedge},
$$

where the right-hand side is the completion of $D(H, K)$ with respect to $\mu_{r_{0}}$. Next, choose $r \in\left(r_{0}, 1\right) \cap p^{\mathbb{Q}}$ such that (3.3.6) holds, and that, in addition, there is $m \in \mathbb{N}$ such that $s=r^{p^{m}}$ satisfies $s>\frac{1}{p}$ and $p^{-1 /(p-1)-1 / e e^{e m}} \leq s^{\varepsilon_{p}}<p^{-1 /(p-1)}$. Here, $e$ is the ramification index of $L$ over $\mathbb{Q}_{p}$, and $q$ is the cardinality of the residue field of $L$. Note that we have $s<r_{0}<r$ and hence a commutative diagram of continuous 
maps:

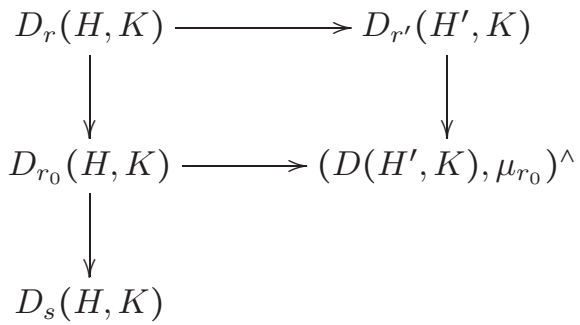

The map $D_{r_{0}}(H, K) \rightarrow\left(D\left(H^{\prime}, K\right), \mu_{r_{0}}\right)^{\wedge}$ is clearly injective, because

$$
\left(D\left(H^{\prime}, K\right), \mu_{r_{0}}\right)^{\wedge}=\bigoplus_{h \in H^{\prime} / H} \delta_{h} \cdot D_{r_{0}}(H, K) .
$$

Moreover, by definition of the norms $\bar{q}_{r}$ we have

$$
D_{r}(H, K)=\bigoplus_{h \in H / H_{0}} \delta_{h} \cdot D_{r}\left(H_{0}, K\right) .
$$

Because $r$ and $s$ are as in Proposition 5.1.1 the map $D_{r}\left(H_{0}, K\right) \rightarrow D_{s}\left(H_{0}, K\right)$ is injective (cf. Proposition 5.2.1). Therefore, it follows from the remark just made that the map $D_{r}(H, K) \rightarrow D_{s}(H, K)$ is injective as well. This implies that the map $D_{r}(H, K) \rightarrow D_{r_{0}}(H, K)$ must be injective as well. Hence the composite map (cf. the diagram above)

$$
D_{r}(H, K) \longrightarrow D_{r_{0}}(H, K) \longrightarrow\left(D\left(H^{\prime}, K\right), \mu_{r_{0}}\right)^{\wedge}
$$

is injective. Hence we see that the map $D_{r}(H, K) \longrightarrow D_{r^{\prime}}\left(H^{\prime}, K\right)$ must be injective as well. We have chosen $r^{\prime}$ such that $D_{r^{\prime}}\left(H^{\prime}, K\right)$ is an integral domain. All maps under consideration are ring homomorphisms, and so $D_{r}(H, K)$ is an integral domain as well.

(ii) We note that in 3.3 .3 we have defined $U_{w, 0}^{-}$in such a way that it is $L$-uniform. Hence the assertion follows immediately from (i).

Corollary 3.3.8. There is a sequence $\left(r_{m}\right)_{m}$ of numbers in $\left(\frac{1}{p}, 1\right) \cap p^{\mathbb{Q}}$, and converging to 1 , such that the following holds for any $m$ : any non-zero left ideal $I$ of $D_{r_{m}}\left(U_{w}^{-}, K\right)$ has non-zero intersection with $U_{r_{m}}\left(\mathfrak{u}_{w}^{-}, U_{w, 0}^{-}\right)$.

Proof. In 3.3 .3 the lattice $p^{t} \Lambda_{1} \subset \mathfrak{u}_{w}^{-}$has been defined so as to assure that $U_{w, 0}^{-}=$ $\exp \left(p^{t} \Lambda_{1}\right)$ is $L$-uniform (cf. Remark 2.2.5). Using Proposition 3.3.5 we can apply Corollary 2.3.4 whose assertion is exactly the claim made above.

\subsection{Modules for the completed distribution algebras.}

3.4.1. The modules $M^{w}(\rho)$ and $m^{w}(\rho)$. Let

$$
\rho: \mathbf{P} \longrightarrow \mathbf{M} \longrightarrow \mathrm{GL}(V)
$$

be the fixed locally analytic representation of $\mathbf{P}$ on $V$ (equipped with the unique Hausdorff locally convex topology) from 3.1.2. The representation of $P_{w}^{+}$which we get by restriction and conjugation will be denoted by $\left(\rho^{w}, V_{w}\right)$, cf. 3.2.3. Consider its (strong) dual $V_{w}^{\prime}:=\left(V_{w}\right)_{b}^{\prime}$, which is a $D\left(P_{w}^{+}, K\right)$-module. We recall our convention to denote Lie algebras of locally analytic groups by their corresponding gothic letters:

$$
\mathfrak{p}_{w}^{+}=\operatorname{Lie}\left(P_{w}^{+}\right)=w \operatorname{Lie}\left(P^{+}\right) w^{-1}, \mathfrak{u}_{w}^{-}=\operatorname{Lie}\left(U_{w}^{-}\right)=w \operatorname{Lie}\left(\mathbf{U}^{-}\right) w^{-1}, \mathfrak{s}=\operatorname{Lie}(\mathbf{S}) .
$$


Via the embedding $U\left(\mathfrak{p}_{w}^{+}\right) \hookrightarrow D\left(P_{w}^{+}, K\right)$ we view $V_{w}^{\prime}$ as a Lie algebra representation of $\mathfrak{p}_{w}^{+}$. We put

$$
m^{w}(\rho):=U(\mathfrak{g}) \otimes_{U\left(\mathfrak{p}_{w}^{+}\right)} V_{w}^{\prime} \cong U\left(\mathfrak{u}_{w}^{-}\right)_{K} \otimes_{K} V_{w}^{\prime} .
$$

Next, consider the induced locally analytic representation $\operatorname{Ind}_{P_{w}^{+}}^{I}\left(\rho^{w}\right)$ and let

$$
M^{w}(\rho)=\left(\operatorname{Ind}_{P_{w}^{+}}^{I}\left(\rho^{w}\right)\right)_{b}^{\prime}=D(I, K) \otimes_{D\left(P_{w}^{+}, K\right)} V_{w}^{\prime}
$$

be the corresponding $D(I, K)$-module. By Proposition 3.3.4 there is a canonical isomorphism of $D\left(U^{-}, K\right)$-modules

$$
M^{w}(\rho)=D(I, K) \otimes_{D\left(P_{w}^{+}, K\right)} V_{w}^{\prime} \simeq D\left(U_{w}^{-}, K\right) \otimes_{K} V_{w}^{\prime} .
$$

Thus we see that the natural map $m^{w}(\rho) \rightarrow M^{w}(\rho)$ is injective.

Proposition 3.4.2. (i) For $r \in\left(\frac{1}{p}, 1\right)$ sufficiently close to 1 the canonical map

$$
V_{w}^{\prime} \longrightarrow D_{r}\left(P_{w}^{+}, K\right) \otimes_{D\left(P_{w}^{+}, K\right)} V_{w}^{\prime}, v \mapsto 1 \otimes v,
$$

is an isomorphism of $K$-vector spaces. Consequently, via this isomorphism, we can extend the continuous operation of $D\left(P_{w}^{+}, K\right)$ on $V_{w}^{\prime}$ to a continuous operation of $D_{r}\left(P_{w}^{+}, K\right)$ on $V_{w}^{\prime}$.

(ii) When the finitely generated $D_{r}(I, K)$-module $D_{r}(I, K) \otimes_{D_{r}\left(P_{w}^{+}, K\right)} V_{w}^{\prime}$ is equipped with its natural topology, the canonical map

$$
D_{r}\left(U_{w}^{-}, K\right) \otimes_{K} V_{w}^{\prime} \longrightarrow D_{r}(I, K) \otimes_{D_{r}\left(P_{w}^{+}, K\right)} V_{w}^{\prime}
$$

is a topological isomorphism.

Proof. (i) The $K$-vector space $V_{w}^{\prime}$ is finite-dimensional and hence finitely generated as $D\left(P_{w}^{+}, K\right)$-module. Thus $V_{w}$ is a strongly admissible representation of $P_{w}^{+}$in the sense of [ST1], $\S 3$. This implies that $V_{w}^{\prime}$ is a co-admissible $D\left(P_{w}^{+}, K\right)$-module in the sense of [ST2], §3 (cf. ST2], paragraph following Proposition 6.4). Hence

$$
\phi: V_{w}^{\prime} \longrightarrow \lim _{r<1}\left(D_{r}\left(P_{w}^{+}, K\right) \otimes_{D\left(P_{w}^{+}, K\right)} V_{w}^{\prime}\right)
$$

is an isomorphism of $K$-vector spaces. By ST2, Theorem A, p. 152, for every $r \in\left(\frac{1}{p}, 1\right)$, the canonical map

$$
\phi_{r}: V_{w}^{\prime} \longrightarrow D_{r}\left(P_{w}^{+}, K\right) \otimes_{D\left(P_{w}^{+}, K\right)} V_{w}^{\prime}
$$

has dense image, and is therefore surjective. For $r_{1}<r_{2}<1$ the map $\phi_{r_{1}}$ factors through $\phi_{r_{2}}$. This implies that the family $\operatorname{ker}\left(\phi_{r}\right)$ is decreasing with increasing $r$. The intersection of all $\operatorname{ker}\left(\phi_{r}\right)$ is the zero space because $\phi$ is injective. As $V_{w}^{\prime}$ is finitedimensional, there must be some $r \in\left(\frac{1}{p}, 1\right)$ such that $\operatorname{ker}\left(\phi_{r}\right)$ is zero. Hence, there is necessarily some $r \in\left(\frac{1}{p}, 1\right)$ such that the map $V_{w}^{\prime} \longrightarrow D_{r}\left(P_{w}^{+}, K\right) \otimes_{D\left(P_{w}^{+}, K\right)} V_{w}^{\prime}$ is bijective.

(ii) By Proposition 3.3.4 it follows that the map in (ii) is a bijection. If we give the right-hand side its natural $D_{r}(I, K)$-module topology, i.e., the quotient topology induced by an arbitrary surjection of $D_{r}(I, K)$-modules

$$
D_{r}(I, K)^{m} \rightarrow D_{r}(I, K) \otimes_{D_{r}\left(P_{w}^{+}, K\right)} V_{w}^{\prime},
$$

then the map in (ii) is a continuous bijective map, and hence, by the open mapping theorem, a homeomorphism. 
3.4.3. The modules $M_{r}^{w}(\rho)$ and $m_{r}^{w}(\rho)$. For the rest of this section, $r<1$ denotes a real number sufficiently close to 1 such that the assertions of Proposition 3.4 .2 and Corollary 3.3 .8 do hold. We put

$$
M_{r}^{w}(\rho):=D_{r}(I, K) \otimes_{D(I, K)} M^{w}(\rho),
$$

which we consider as a module over the Banach algebra $D_{r}(I, K)$. By the foregoing proposition, we have

$$
\begin{aligned}
D_{r}(I, K) \otimes_{D(I, K)} M^{w}(\rho) & =D_{r}(I, K) \otimes_{D\left(P_{w}^{+}, K\right)} V_{w}^{\prime} \\
& =D_{r}(I, K) \otimes_{D_{r}\left(P_{w}^{+}, K\right)}\left(D_{r}\left(P_{w}^{+}, K\right) \otimes_{D\left(P_{w}^{+}, K\right)} V_{w}^{\prime}\right) \\
& =D_{r}(I, K) \otimes_{D_{r}\left(P_{w}^{+}, K\right)} V_{w}^{\prime} .
\end{aligned}
$$

Using this and Proposition 3.3 .4 we see that the map

$$
D_{r}\left(U_{w}^{-}, K\right) \otimes_{K} V_{w}^{\prime} \longrightarrow M_{r}^{w}(\rho)
$$

is an isomorphism of $D_{r}\left(U_{w}^{-}, K\right)$-modules. Therefore, the natural map

$$
M^{w}(\rho) \longrightarrow M_{r}^{w}(\rho)
$$

is an embedding. We denote the topological closure of $m^{w}(\rho)$ in $M_{r}^{w}(\rho)$ by $m_{r}^{w}(\rho)$. Because $U_{r}\left(\mathfrak{u}_{w}^{-}, U_{w, 0}^{-}\right)$is the topological closure of $U\left(\mathfrak{u}_{w}^{-}\right)_{K}$ in $D_{r}\left(U_{w}^{-}, K\right)$ we have

$$
m_{r}^{w}(\rho)=U_{r}\left(\mathfrak{u}_{w}^{-}, U_{w, 0}^{-}\right) \otimes_{K} V_{w}^{\prime} .
$$

Since the maximal split torus $\mathbf{S}$ is contained in $\mathbf{P}$, we have a natural diagonal action of $U(\mathfrak{s})$ on $m^{w}(\rho)$.

Lemma 3.4.4. (i) For any $\mathfrak{x} \in \mathfrak{s}$ the action of $\mathfrak{x}$ on $m^{w}(\rho)$ is continuous with respect to the topology on $m_{r}^{w}(\rho)$, and hence extends continuously to $m_{r}^{w}(\rho)$.

(ii) The $U(\mathfrak{s})$-module structure on $m^{w}(\rho)$ extends to a $U(\mathfrak{s})$-module structure on $m_{r}^{w}(\rho)$.

(iii) For any $\lambda \in \mathfrak{s}^{*}$ the weight space $m_{r}^{w}(\rho)_{\lambda}$ is finite-dimensional.

Proof. We begin by considering again the way we have defined the subgroup $U_{w, 0}^{-}$ in 3.3.3. Recall that we started with an $L$-uniform (normal) subgroup $H$ of $G$, and a lattice $\Lambda \subset \operatorname{Lie}(H)$ on which $\exp _{H}$ is defined and maps $\Lambda$ bijectively to $H$. Then we have chosen $e$ large enough such that $U_{x_{0}}^{(e)}$ is contained in $H$. Then we have put

$$
\Lambda_{1}:=\exp _{H}^{-1}\left(U_{x_{0}}^{(e)} \cap w \mathbf{U}^{-} w^{-1}\right)=\operatorname{Ad}(w)\left(\exp _{H}^{-1}\left(U_{x_{0}}^{(e)} \cap \mathbf{U}^{-}\right)\right)
$$

and $U_{w, 0}^{-}=\exp _{H}\left(p^{t} \Lambda_{1}\right)$ for $t \gg 0$.

Our aim is to show that $\Lambda_{1}$ has an $\mathfrak{o}_{L}$-basis consisting of root elements.

By [S-S], Proposition I.2.7, we have

$$
U_{x_{0}}^{(e)} \cap \mathbf{U}^{-}=\prod_{\alpha \in\left(\Phi^{-} \backslash \Phi_{\mathbf{P}}\right) \cap \Phi^{\text {red }}}\left(U_{f_{x_{0}}^{*}+e} \cap U_{\alpha}\right)
$$

where $\Phi_{\mathbf{P}}$ is the root system of the Levi subgroup of $\mathbf{P}$ which contains the torus $\mathbf{S}$, and where

$$
U_{f_{x_{0}}^{*}+e} \cap U_{\alpha}=U_{\alpha, f_{x_{0}}^{*}(\alpha)+e} \cdot U_{2 \alpha, 2 f_{x_{0}}^{*}(\alpha)+e} \cdot
$$


We refer to [S-S] (and [BT2]) for the definitions of the various groups appearing here. We want to show that

$$
\begin{aligned}
& \exp _{H}^{-1}\left(U_{f_{x_{0}}^{*}+e} \cap U_{\alpha}\right) \\
& =\left(\exp _{H}^{-1}\left(U_{f_{x_{0}}^{*}+e} \cap U_{\alpha}\right) \cap \mathfrak{g}_{\alpha}\right) \oplus\left(\exp _{H}^{-1}\left(U_{f_{x_{0}}^{\star}+e} \cap U_{\alpha}\right) \cap \mathfrak{g}_{2 \alpha}\right) .
\end{aligned}
$$

Here $\mathfrak{g}_{\alpha}, \mathfrak{g}_{2 \alpha}$ denote, as usual, the root subspaces 6 By identity (3.4.5) it follows that (3.4.6) holds if we have

$$
\begin{aligned}
& \exp _{H}^{-1}\left(U_{\alpha, f_{x_{0}}^{*}(\alpha)+e}\right) \\
& \quad=\left(\exp _{H}^{-1}\left(U_{\alpha, f_{x_{0}}^{*}(\alpha)+e}\right) \cap \mathfrak{g}_{\alpha}\right) \oplus\left(\exp _{H}^{-1}\left(U_{\alpha, f_{x_{0}}^{*}(\alpha)+e}\right) \cap \mathfrak{g}_{2 \alpha}\right) .
\end{aligned}
$$

To show that (3.4.7) holds is a non-trivial problem only if $2 \alpha$ is a root, which we assume from now on. At this point we have to consider the actual definition of the groups involved, and this means to trace through the ouvre of Bruhat and Tits. We will give the reader only some guidelines where to look in BT2. By BT2, 5.1 .16 , one can easily reduce the problem to the case when $\mathfrak{G}$ is quasi-split. By BT2, 4.3.5, one has $U_{\alpha, k}=\mathfrak{U}_{\alpha, k}\left(\mathfrak{o}_{L}\right)$, and this group has an explicit description in terms of a group denoted $H\left(L, L_{2}\right)$ in [BT2], cf. [BT2], 4.1.9, 4.1.15] But the description of $H\left(L, L_{2}\right)$ given in BT2, 4.1.15, shows that 3.4.7) does indeed hold.

Hence we see that $\exp _{H}^{-1}\left(U_{x_{0}}^{(e)} \cap \mathbf{U}^{-}\right)$has an $\mathfrak{o}_{L}$-basis consisting of weight vectors, and the same is therefore true for the lattice $\Lambda_{1}$ and for $p^{t} \Lambda_{1}$. Thus, let $\left(\mathfrak{X}_{1}, \ldots, \mathfrak{X}_{d}\right)$ be an $\mathfrak{o}_{L}$-basis of $p^{t} \Lambda_{1}=\exp _{H}^{-1}\left(U_{w, 0}^{-}\right)$which consists of weight vectors $\mathfrak{X}_{i} \in \mathfrak{g}_{\alpha_{i}}$.

(i) Because $U_{w, 0}^{-}$is $L$-uniform, we can apply part (ii) of Kohlhaase's Theorem 2.3 .2 to the basis $\left(\mathfrak{X}_{1}, \ldots, \mathfrak{X}_{d}\right)$ : the closure $U_{r}\left(\mathfrak{u}_{w}^{-}, U_{w, 0}^{-}\right)$of $U\left(\mathfrak{u}_{w}^{-}\right) \otimes_{L} K$ in $D_{r}\left(U_{w, 0}^{-}, K\right)$ (cf. 2.3.1) consists exactly of those series

$$
\sum_{n \in \mathbb{N}_{0}^{d}} d_{n} \mathfrak{X}^{n}
$$

for which

$$
\lim _{|n| \rightarrow \infty}\left|d_{n}\right| \nu_{r}\left(\mathfrak{X}^{n}\right)=0 .
$$

Here, $\nu_{r}$ is a norm on $U_{r}\left(\mathfrak{u}_{w}^{-}, U_{w, 0}^{-}\right)$which is equivalent to $\bar{q}_{r}$ and $\nu_{r}\left(\sum_{n} d_{n} \mathfrak{X}^{n}\right)=$ $\sup _{n}\left|d_{n}\right| \nu_{r}\left(\mathfrak{X}^{n}\right)$. Let $v_{1}, \ldots, v_{k}$ be a basis of $V_{w}^{\prime}$ which consists of weight vectors: $\mathfrak{x} \cdot v_{j}=\gamma_{j}(\mathfrak{x}) v_{j}$ for all $\mathfrak{x} \in \mathfrak{s}, 1 \leq j \leq k$. Then we have for any $\mathfrak{x} \in \mathfrak{s}$ :

$$
\mathfrak{x} \cdot\left(\mathfrak{X}^{n} \otimes v_{j}\right)=\left(\gamma_{j}+\sum_{1 \leq i \leq k} n_{i} \alpha_{i}\right)(\mathfrak{x}) \cdot\left(\mathfrak{X}^{n} \otimes v_{j}\right) .
$$

It is obvious that there is $C>0$ (depending on $\mathfrak{x}$ ) such that for all $j \in\{1, \ldots, k\}$ and all $n \in \mathbb{N}_{0}^{d}$ one has

$$
\left|\left(\gamma_{j}+\sum_{1 \leq i \leq k} n_{i} \alpha_{i}\right)(\mathfrak{x})\right|_{K} \leq C .
$$

This shows that the action of $\mathfrak{x}$ on $m^{w}(\rho)$ extends to a continuous endomorphism of $m_{r}^{w}(\rho)$.

(ii) Is an immediate consequence of (i).

\footnotetext{
${ }^{6}$ Note that, according to the conventions used in [S-S] and [BT2], one has $\operatorname{Lie}\left(U_{\alpha}\right)=\mathfrak{g}_{\alpha} \oplus \mathfrak{g}_{2 \alpha}$.

${ }^{7}$ The field denoted $L$ in [BT2], ch. 4 , is not the same as our field $L$; our field $L$ is the field $K$ in BT2, ch. 4.
} 
(iii) In (i) we have seen that the elements $\mathfrak{X}^{n} \otimes v_{j} \in m_{r}^{w}(\rho)$ are weight vectors for the action of $\mathfrak{s}$. If

$$
\mu=\sum_{n \in \mathbb{N}_{0}^{d}, 1 \leq j \leq k} d_{n, j} \mathfrak{X}^{n} \otimes v_{j} \in m_{r}^{w}(\rho)=U_{r}\left(U_{0}^{-}, K\right) \otimes_{K} V_{w}^{\prime}
$$

is an element of $m_{r}^{w}(\rho)_{\lambda}$, then

$$
\lambda=\gamma_{j}+\sum_{1 \leq i \leq k} n_{i} \alpha_{i}
$$

if $d_{n, j} \neq 0$. This is an immediate consequence of the fact that the monomials $\left\{\mathfrak{X}^{n}\right\}_{n \in \mathbb{N}_{0}^{d}}$ are an orthogonal basis of $U_{r^{\prime}}\left(U_{0}^{-}, K\right)$ with respect to the norm $\nu_{r}$, cf. Theorem 2.3.2. Because the characters $\alpha_{i}$ do all occur in the Lie algebra $\mathfrak{u}_{w}^{-}$, there can only be finitely many possibilities to write $\lambda$ as a sum as above. Therefore, the weight spaces $m_{r}^{w}(\rho)$ are all finite-dimensional.

From $m_{r}^{w}(\rho)$ back to $m^{w}(\rho)$. From the result above we can deduce that closed $U(\mathfrak{s})$-invariant subspaces of $m_{r}^{w}(\rho)$ are in bijection with $U(\mathfrak{s})$-invariant subspaces of $m^{w}(\rho)$.

Proposition 3.4.8. (i) We have an inclusion preserving bijection

$$
\begin{aligned}
\left\{\begin{array}{c}
\text { closed } U(\mathfrak{s}) \text {-invariant } \\
\text { subspaces of } m_{r}^{w}(\rho)
\end{array}\right\} & \stackrel{\sim}{\longrightarrow}\left\{U(\mathfrak{s}) \text {-invariant subspaces of } m^{w}(\rho)\right\} \\
W & \longmapsto W \cap m^{w}(\rho) .
\end{aligned}
$$

Any $U(\mathfrak{s})$-invariant subspace $W$ of $m_{r}^{w}(\rho)$ is the direct sum of its weight components: $W=\oplus W_{\lambda}$.

(ii) Let $N \subset m_{r}^{w}(\rho)$ be a closed $U(\mathfrak{s})$-invariant subspace, $N \neq 0$. Then $N \cap$ $m^{w}(\rho) \neq 0$. In particular, any weight vector for the action of $\mathfrak{s}$ lies already in $m^{w}(\rho)$.

Proof. (i) This statement follows from Lemma 3.4.4 and [Fe] 1.3.12.

(ii) This is an immediate consequence of (i).

Lemma 3.4.9. There is $r^{\prime} \in(0,1)$ such that $U\left(\mathfrak{u}_{w}^{-}\right) \otimes_{L} K$ is dense in $D_{r^{\prime}}\left(U_{w, 0}^{-}, K\right)$.

Proof. Let $\left(\mathfrak{x}_{i}\right)_{i}$ be a basis of the $\mathbb{Z}_{p}$-Lie algebra $p^{t} \Lambda_{1}$ of $U_{w, 0}^{-}$, with the notation as introduced in sec. 3.3.3. Then the elements $\left(\exp \left(\mathfrak{x}_{i}\right)\right)_{i}$, for an arbitrary but fixed ordering, form a $p$-basis of $U_{w, 0}^{-}$. Put $b_{i}=\exp \left(\mathfrak{x}_{i}\right)-1 \in D\left(R_{\mathbb{Q}_{p}}^{L} U_{w, 0}^{-}, K\right)$, so that the monomials $\mathbf{b}^{n}$ in the $b_{i}$ form an orthogonal basis of $D_{r}\left(R_{\mathbb{Q}_{p}}^{L} U_{w, 0}^{-}, K\right)$, as in sec. 2.2.6. For $0<r^{\prime}<1$ sufficiently close to 0 the right-hand side of the identity $b_{i}=\exp \left(\mathfrak{x}_{i}\right)-1$ can be expanded as a convergent series

$$
\sum_{\nu \geq 1} \frac{\mathfrak{x}_{i}^{\nu}}{\nu !}
$$

in $D_{r}\left(R_{\mathbb{Q}_{p}}^{L} U_{w, 0}^{-}, K\right)$. This shows that we can approximate $b_{i}$ by elements in $U\left(\mathfrak{u}_{w}^{-}\right)_{K}$. Hence $U\left(\mathfrak{u}_{w}^{-}\right) \otimes_{L} K$ is dense in $D_{r^{\prime}}\left(U_{w, 0}^{-}, K\right)$.

Proposition 3.4.10. Every non-trivial $U(\mathfrak{s})$-invariant $D_{r}\left(U_{w}^{-}, K\right)$-submodule of $M_{r}^{w}(\rho)$ has a non-trivial intersection with $m_{r}^{w}(\rho)$.

Proof. (See also Proposition 11 of $\left[\mathrm{Fr}\right.$.) Fix a basis $v_{1}, \ldots, v_{k}$ of weight vectors in $V_{w}^{\prime}$ with respect to the action of $U(\mathfrak{s})$. We obtain $D_{r}\left(U_{w}^{-}, K\right)$-submodules

$$
D_{r}\left(U_{w}^{-}, K\right) \otimes K v_{i} \subset M_{r}^{w}(\rho)
$$


which are $U(\mathfrak{s})$-invariant. Consider the projections

$$
\operatorname{pr}_{i}: M_{r}^{w}(\rho)=D_{r}\left(U_{w}^{-}, K\right) \otimes_{K} V_{w}^{\prime} \rightarrow D_{r}\left(U_{w}^{-}, K\right) \otimes_{K} K v_{i}
$$

and let $N \subset M_{r}^{w}(\rho)$ be a $D_{r}\left(U_{w}^{-}, K\right)$ submodule which is $U(\mathfrak{s})$-invariant. By defining

$$
N^{(i)}=\bigcap_{1 \leq j<i} \operatorname{ker}\left(\operatorname{pr}_{j}\right) \cap N,
$$

we obtain a descending filtration of $D_{r}\left(U_{w}^{-}, K\right) \times U(\mathfrak{s})$-modules

$$
0=N^{(k+1)} \subset N^{(k)} \subset \ldots \subset N^{(2)} \subset N^{(1)}=N .
$$

Let $1 \leq i \leq k$ be the unique index with

$$
0=N^{(i+1)} \subset N^{(i)} \neq 0 .
$$

Identifying $D_{r}\left(U_{w}^{-}, K\right) \otimes_{K} K v_{i}$ with $D_{r}\left(U_{w}^{-}, K\right)$ as $D_{r}\left(U_{w}^{-}, K\right)$-modules we see by Corollary 3.3.8 that

$$
\operatorname{pr}_{i}\left(N^{(i)}\right) \cap\left(U_{r}\left(\mathfrak{u}_{w}^{-}, U_{w, 0}^{-}\right) \otimes_{K} K v_{i}\right) \neq 0 .
$$

By applying Proposition 3.4 .8 we can infer that

$$
\operatorname{pr}_{i}\left(N^{(i)}\right) \cap\left(U\left(\mathfrak{u}_{w}^{-}\right) \otimes_{L} K v_{i}\right) \neq 0 .
$$

Therefore, there is an element $F \in N^{(i)}$ such that $\operatorname{pr}_{i}(F) \in U\left(\mathfrak{u}_{w}^{-}\right) \otimes_{L} K v_{i}$ is a weight vector (again by Proposition 3.4.8). In order to prove the statement of our proposition, it suffices to show that $\operatorname{pr}_{j}(F) \in U_{r}\left(\mathfrak{u}_{w}^{-}, U_{w, 0}^{-}\right) \otimes_{K} K v_{j}, j=i+1, \ldots, k$. Suppose that there is an index $i<j \leq k$ such that $\operatorname{pr}_{j}(F) \notin U_{r}\left(\mathfrak{u}_{w}^{-}, U_{w, 0}^{-}\right) \otimes_{K} K v_{j}$.

We want to show that $\operatorname{pr}_{j}(F)$ cannot be a weight vector for the action of $U(\mathfrak{s})$.

To this end, choose $r^{\prime} \in(0, r)$ sufficiently small such that $U\left(\mathfrak{u}_{w}^{-}\right) \otimes_{L} K$ is dense in $D_{r^{\prime}}\left(U_{w, 0}^{-}, K\right)$, cf. Lemma 3.4.9, Then we have a commutative diagram of embeddings

$$
\begin{array}{lll}
U_{r^{\prime}}\left(\mathfrak{u}_{w}^{-}, U_{w, 0}^{-}\right) \otimes_{K} K v_{j} & \stackrel{D_{r^{\prime}}}{ }\left(U_{w, 0}^{-}, K\right) \otimes_{K} K v_{j} \\
\cup & \cup \\
U_{r}\left(\mathfrak{u}_{w}^{-}, U_{w, 0}^{-}\right) \otimes_{K} K v_{j} & \rightarrow & D_{r}\left(U_{w, 0}^{-}, K\right) \otimes_{K} K v_{j}
\end{array}
$$

Therefore, we can consider $\operatorname{pr}_{j}(F)$ as an element of $U_{r^{\prime}}\left(\mathfrak{u}_{w}^{-}, U_{w, 0}^{-}\right) \otimes_{K} K v_{j}$. If $\operatorname{pr}_{j}(F)$ was a weight vector, it would then automatically be an element of $U\left(\mathfrak{u}_{w}^{-}\right) \otimes_{L} K v_{j}$, by Proposition 3.4 .8 (ii). Hence, a fortiori, $\operatorname{pr}_{j}(F)$ would be in $U_{r}\left(\mathfrak{u}_{w}^{-}, U_{w, 0}^{-}\right) \otimes_{K} K v_{j}$.

Thus we have shown that $\operatorname{pr}_{j}(F)$ is not a weight vector. Hence we may choose $\lambda \in U(\mathfrak{s})$ such that $\lambda \cdot \operatorname{pr}_{j}(F)$ is not a scalar multiple of $\operatorname{pr}_{j}(F)$. Let $C_{\lambda} \in K$ be the scalar with

$$
\lambda \cdot \operatorname{pr}_{i}(F)=C_{\lambda} \cdot \operatorname{pr}_{i}(F) .
$$

Then the non-zero element $\left(\lambda-C_{\lambda}\right) \cdot F$ is contained in $N^{(i+1)}=0$, which is a contradiction.

Corollary 3.4.11. If $m(\rho)=U(\mathfrak{g}) \otimes_{U\left(\mathfrak{p}^{+}\right)} V^{\prime}$ is a simple $U(\mathfrak{g})$-module then:

(i) $m^{w}(\rho)=U(\mathfrak{g}) \otimes_{U\left(\mathfrak{p}_{w}^{+}\right)} V_{w}^{\prime}$ is a simple $U(\mathfrak{g})$-module for every $w$.

(ii) $M_{r}^{w}(\rho)$ is a simple $D_{r}(I, K)$-module for every $w$.

Proof. (i) Note that the map

$$
U(\mathfrak{g}) \otimes_{U\left(\mathfrak{p}^{+}\right)} V^{\prime} \longrightarrow U(\mathfrak{g}) \otimes_{U\left(\mathfrak{p}_{w}^{+}\right)} V_{w}^{\prime}, \mathfrak{z} \otimes v \mapsto \operatorname{ad}(w)(\mathfrak{z}) \otimes v
$$


is an isomorphism of the underlying vector spaces. It sends $U(\mathfrak{g})$-submodules to $U(\mathfrak{g})$-submodules. The left-hand side is therefore a simple $U(\mathfrak{g})$-module if and only if the right-hand side is a simple $U(\mathfrak{g})$-module.

(ii) Let $N \subset M_{r}^{w}(\rho)$ be a $D_{r}(I, K)$-submodule. It is automatically closed because $D_{r}(I, K)$ is noetherian and $M_{r}^{w}(\rho)$ is a finitely generated $D_{r}(I, K)$-module. By the previous proposition, we see that $N \cap m_{r}^{w}(\rho) \neq 0$. By Proposition 3.4.8 we get that $N \cap m^{w}(\rho) \neq 0$. Since $m^{w}(\rho)$ is a simple $U(\mathfrak{g})$-module, we obtain an inclusion $m^{w}(\rho) \subset N$. Thus we conclude that $1 \otimes V_{w}^{\prime}$ is contained in $N$ and therefore $N=M_{r}^{w}(\rho)$.

Theorem 3.4.12. If $m(\rho)=U(\mathfrak{g}) \otimes_{U\left(\mathfrak{p}^{+}\right)} V^{\prime}$ is a simple $U(\mathfrak{g})$-module, then

$$
M^{w}(\rho)=\left(\operatorname{Ind}_{P_{w}^{+}}^{I}\left(\rho^{w}\right)\right)_{b}^{\prime}
$$

is a simple $D(I, K)$-module for every $w$.

Proof. It follows from the definition of $M^{w}(\rho)$ that this is a finitely generated $D(I, K)$-module (using that $V$ is finite-dimensional over $K)$. Therefore, $\operatorname{Ind}_{P_{w}^{+}}^{I}\left(\rho^{w}\right)$ is a strongly admissible representation of $I$ in the sense of [ST1], §3. This implies that $V_{w}^{\prime}$ is a co-admissible $D\left(P_{w}^{+}, K\right)$-module in the sense of [ST2], §3 (cf. [ST2, paragraph following Proposition 6.4). Now we can use [ST2, Lemma 3.9 and Corollary 3.4.11 to conclude that $M^{w}(\rho)$ is a simple $D(I, K)$-module.

3.5. The main result. The last essential step, to show the topological irreducibility of the induced representation

$$
\operatorname{Ind}_{\mathbf{P}}^{\mathbf{G}}(\rho) \cong \operatorname{Ind}_{P^{+}}^{G}(\rho),
$$

is to prove that the various $D(I, K)$-modules $M^{w}(\rho)$ are pairwise non-isomorphic.

Proposition 3.5.1. Every homomorphism $M^{w^{\prime}}(\rho) \rightarrow M^{w}(\rho)$ of $D(I, K)$-modules for $w \neq w^{\prime}$ is zero.

Proof. Our proof is a slight generalization of [Fr], Proposition 12. A homomorphism $M^{w^{\prime}}(\rho) \rightarrow M^{w}(\rho)$ corresponds by duality (cf. 2.4), to a homomorphism

$$
\operatorname{Ind}_{P_{w}^{+}}^{I}\left(\rho^{w}\right) \longrightarrow \operatorname{Ind}_{P_{w^{\prime}}^{+}}^{I}\left(\rho^{w^{\prime}}\right)
$$

of locally analytic $I$-representations. By Frobenius reciprocity this corresponds to a continuous $P_{w^{\prime}}^{+}$-homomorphism

$$
\operatorname{Ind}_{P_{w}^{+}}^{I}\left(\rho^{w}\right) \rightarrow V_{w^{\prime}}=V .
$$

From the decomposition $I=U_{w}^{-} \cdot P_{w}^{+}$we deduce an isomorphism

$$
\operatorname{Ind}_{P_{w}^{+}}^{I}\left(\rho^{w}\right) \stackrel{\simeq}{\longrightarrow} C_{L}^{a n}\left(U_{w}^{-}, V\right)
$$

of representations of $U_{w}^{-} \cap P_{w^{\prime}}^{+}$. Since $w \neq w^{\prime}$ the intersection $w^{\prime} \mathbf{U}\left(w^{\prime}\right)^{-1} \cap w \mathbf{U}^{-} w^{-1}$ contains a root group. Here, $\mathbf{U}$ is the unipotent radical of $\mathbf{P}$. Therefore,

$$
U:=w^{\prime} \mathbf{U}\left(w^{\prime}\right)^{-1} \cap U_{w}^{-} \cap P_{w^{\prime}}^{+}
$$

is a non-trivial $L$-analytic group of positive dimension. The group $w^{\prime} \mathbf{U}\left(w^{\prime}\right)^{-1}$ acts trivially on $V_{w^{\prime}}$, by assumption. Thus $U$ acts trivially on $V_{w^{\prime}}$. A homomorphism

$$
\operatorname{Ind}_{P_{w}^{+}}^{I}\left(\rho^{w}\right) \longrightarrow \operatorname{Ind}_{P_{w^{\prime}}^{+}}^{I}\left(\rho^{w^{\prime}}\right)
$$


therefore gives rise to a continuous map

$$
\phi: C_{L}^{a n}\left(U_{w}^{-}, V\right) \longrightarrow V
$$

which is $U$-equivariant, with $U$ acting trivially on $V$. The canonical projection $U_{w}^{-} \rightarrow U \backslash U_{w}^{-}$has a locally $L$-analytic section, so that we can find an isomorphism of locally $L$-analytic manifolds $U_{w}^{-} \simeq U \times U^{\prime}$ with some compact $L$-analytic manifold $U^{\prime}$. This isomorphism we may assume to be compatible with the action of $U$ by left translation (acting trivially on $U^{\prime}$ ). This in turn gives rise to an isomorphism

$$
C_{L}^{a n}\left(U_{w}^{-}, V\right) \stackrel{\simeq}{\longrightarrow} C_{L}^{a n}(U, K) \hat{\otimes}_{K, \pi} C_{L}^{a n}\left(U^{\prime}, V\right),
$$

cf. ST3, Lemma A.1 and Proposition A.2. Let $\lambda: V \rightarrow K$ be a linear form and fix $g \in C_{L}^{a n}\left(U^{\prime}, V\right)$. The map

$$
\phi_{\lambda, g}: C_{L}^{a n}(U, K) \rightarrow K, f \mapsto \phi_{\lambda, g}(f)=\lambda(\phi(f g))
$$

then has the property that for all $u \in U$,

$$
\phi_{\lambda, g}(x \mapsto f(u x))=\phi_{\lambda, g}(f) .
$$

Because $U$ does not have a $p$-adic Haar measure, there is no non-zero $U$-invariant continuous linear form on $C_{L}^{a n}(U, K)$. Hence, we find that $\phi_{\lambda, g}=0$. The functions of the form $f \cdot g, f \in C_{L}^{a n}(U, K), g \in C_{L}^{a n}\left(U^{\prime}, V\right)$, span a dense subspace of $C_{L}^{a n}\left(U_{w}^{-}, V\right)$. Hence we deduce that $\lambda \circ \phi=0$ for all linear forms $\lambda$ on $V$. This shows that $\phi$ is necessarily zero.

Theorem 3.5.2. Suppose $m(\rho)$ is simple as a $U(\mathfrak{g})$-module. Then

(i) $M(\rho)=\left(\operatorname{Ind}_{P^{+}}^{G}(\rho)\right)_{b}^{\prime}$ is simple as a $D(G, K)$-module. A fortiori, the representation $\operatorname{Ind}_{P^{+}}^{G}(\rho)$ is topologically irreducible.

(ii) $\operatorname{Ind}_{\mathbf{P}}^{\mathbf{G}}(\rho)$ is a topologically irreducible representation of $\mathbf{G}=\mathfrak{G}(L)$.

Proof. Consider the decomposition

$$
\left(\operatorname{Ind}_{P^{+}}^{G}(\rho)\right)_{b}^{\prime}=\bigoplus_{W / W_{\mathbf{P}}} M^{w}(\rho) .
$$

Each term $M^{w}(\rho)$ is by Theorem 3.4 .12 a simple $D(I, K)$-module. Furthermore, the summands are not pairwise isomorphic by the previous proposition. Since the Weyl group permutes the summands transitively, $M(\rho)$ is a simple $D(G, K)$ module. By the relation between $D(G, K)$-modules and representations of $G$, we conclude that $\operatorname{Ind}_{P^{+}}^{G}(\rho)$ is a topologically irreducible representation of $G$, cf. [2.4] The second assertion follows immediately from (i).

\section{EXAmples}

4.1. Irreducibility of Verma modules. Theorem 3.5 .2 is of course only useful if one can determine whether the generalized Verma module $m(\rho)=U(\mathfrak{g}) \otimes_{U(\mathfrak{p})} \rho^{\prime}$ is irreducible or not. To simplify this problem, one may pass to a finite extension $\tilde{K}$ of $K$ such that the Lie algebra

$$
\mathfrak{g}_{\tilde{K}}=\mathfrak{g} \otimes_{L} \tilde{K}
$$

is split over $\tilde{K}$. Clearly, if $m(\rho) \otimes_{K} \tilde{K}$ is irreducible as a module over $U\left(\mathfrak{g}_{\tilde{K}}\right)$, then $m(\rho)$ is simple as a $U(\mathfrak{g})$-module. If one can reduce the problem to a question about classical Verma modules, i.e., those which are induced from one-dimensional 
representations of a Borel subalgebra, one is in a particular simple situation. In the next paragraph we recall the setup from [D], Ch.7.

Suppose $\mathfrak{g}$ is a split reductive Lie algebra over $K$. Let $\mathfrak{h}$ be Cartan subalgebra of $\mathfrak{g}, \mathfrak{b}$ be a Borel subalgebra containing $\mathfrak{h}, \Phi$ the root system of $\mathfrak{g}$ with respect to $\mathfrak{h}$, and $\Phi^{+} \supset \Delta$ the set of positive and simple roots, respectively. Denote by $\delta=\frac{1}{2} \sum_{\alpha \in \Phi^{+}} \alpha$ the sum of positive roots, as usual. Let $\left\{X_{\beta}, \beta \in \Phi ; H_{\alpha}, \alpha \in \Delta\right\}$ be a Chevalley basis of $\mathfrak{g}_{\text {der }}=[\mathfrak{g}, \mathfrak{g}]$. For a character $\lambda \in \operatorname{Hom}_{K}(\mathfrak{h}, K)$, let $K_{\lambda}$ be the one-dimensional representation of $\mathfrak{h}$ on $K$ defined by $\lambda$ and extend this to a representation of $\mathfrak{b}$ by letting the nilpotent radical act trivially.

Theorem 4.1.1 (Bernstein, Gelfand, Gelfand). The Verma module $U(\mathfrak{g}) \otimes_{U(\mathfrak{b})} K_{\lambda}$ is a simple $U(\mathfrak{g})$-module if and only if $(\lambda+\delta)\left(H_{\alpha}\right) \notin \mathbb{Z}_{>0}$ for all $\alpha \in \Delta$.

We refer to $[\mathrm{D}$, Theorem 7.6.24. Note that Dixmier uses the normalized induction, i.e., his $\lambda$ is our $\lambda+\delta$.

Example 4.1.2. Let $\mathfrak{G}=\mathrm{GL}_{2, L}$ be the group $\mathrm{GL}_{2}$ over $L, \mathfrak{P}$ the Borel subgroup of upper triangular matrices, $\mathfrak{S} \subset \mathfrak{P}$ the diagonal torus and

$$
\chi: \mathbf{S}=\mathfrak{S}(L) \rightarrow K^{*},\left(\begin{array}{cc}
t_{1} & 0 \\
0 & t_{2}
\end{array}\right) \mapsto \chi_{1}\left(t_{1}\right) \chi_{2}\left(t_{2}\right),
$$

where the characters $\chi_{i}: L^{*} \rightarrow K^{*}, i=1,2$, are locally $L$-analytic. We lift $\chi$ to a character of $\mathbf{P}=\mathfrak{P}(L)$, and denote by $K_{\chi}$ the corresponding representation on $K$. Consider the locally $L$-analytic representation $\operatorname{Ind}_{\mathbf{P}}^{\mathbf{G}}\left(K_{\chi}\right)$. For $t \in L^{*}$ close to 1 , one has $\chi_{i}(t)=t^{c_{i}}$ with $c_{i}=\frac{d}{d t} \chi_{i}(1) \in K, i=1,2$. The representation of $\mathfrak{p}$ on the dual space $\left(K_{\chi}\right)^{\prime} \simeq K$ is given by the linear form

$$
\left(\begin{array}{cc}
t_{1} & * \\
0 & t_{2}
\end{array}\right) \mapsto-\left(c_{1} t_{1}+c_{2} t_{2}\right) \text {. }
$$

By Theorems 4.1 .1 and 3.5 .2 we conclude that $\operatorname{Ind}_{\mathbf{P}}^{\mathbf{G}}\left(K_{\chi}\right)$ is topologically irreducible if $-\left(c_{1}-c_{2}\right) \notin \mathbb{Z}_{\geq 0}$. This was shown also in [KS, cf. Theorem 3.1.6.

4.2. Restriction of scalars. Let $\tilde{L}$ be a finite extension of $L$ and denote by $\Gamma$ the set of $L$-embeddings of $\tilde{L}$ into $K$. Suppose that the cardinality of $\Gamma$ is equal to $[\tilde{L}: L]$. Let $\tilde{\mathfrak{G}}$ be a connected split reductive group over $\tilde{L}, \tilde{\mathfrak{S}}$ a maximal split torus and $\tilde{\mathfrak{P}}$ a Borel subgroup containing $\tilde{\mathfrak{S}}$. Then we consider the reductive group $\mathfrak{G}=\operatorname{Res}_{L}^{\tilde{L}} \tilde{\mathfrak{G}}$ over $L$. Put $\mathfrak{P}=\operatorname{Res}_{L}^{\tilde{L}} \tilde{\mathfrak{P}}, \mathfrak{T}=\operatorname{Res}_{L}^{\tilde{L}} \tilde{\mathfrak{S}}$, and

$$
\mathbf{G}=\mathfrak{G}(L)=\tilde{\mathfrak{G}}(\tilde{L}), \mathbf{P}=\mathfrak{P}(L)=\tilde{\mathfrak{P}}(\tilde{L}), \mathbf{T}=\mathfrak{T}(L)=\tilde{\mathfrak{S}}(\tilde{L}) .
$$

Consider a locally $L$-analytic character $\chi: \mathbf{P} \rightarrow K^{*}$ which factors through $\mathbf{T}$, and let $d \chi \in \operatorname{Hom}_{L}(\mathfrak{t}, K)$ be the derivative of $\chi$. Because $\mathfrak{t}=\mathfrak{s} \otimes_{L} \tilde{L}$ and by our assumption on $K$ we have a canonical $K$-linear isomorphism

$$
\operatorname{Hom}_{L}(\mathfrak{t}, K)=\operatorname{Hom}_{L}(\mathfrak{s}, K) \otimes_{K} \operatorname{Hom}_{L}(\tilde{L}, K) .
$$

The set $\Gamma$ is a basis for the set of $L$-vector space homomorphism $\operatorname{Hom}_{L}(\tilde{L}, K)$, and so we can write

$$
d \chi=\sum_{\sigma \in \Gamma} d \chi_{\sigma} \otimes \sigma
$$

with uniquely determined $L$-linear maps $d \chi_{\sigma}: \mathfrak{s} \rightarrow K$.

The proof of the following assertion is an easy exercise: 
Proposition 4.2.1. (i) The map

$$
\tilde{L} \otimes_{L} K \longrightarrow \prod_{\sigma \in \Gamma} K, \xi \otimes \zeta \mapsto(\sigma(\xi) \zeta)_{\sigma},
$$

induces an isomorphism $U(\mathfrak{g}) \otimes_{L} K \simeq \prod_{\sigma \in \Gamma} U\left(\tilde{\mathfrak{g}}_{K}\right)$ such that the $U(\mathfrak{g}) \otimes_{L} K$-module $U\left(\mathfrak{g}_{K}\right) \otimes_{U\left(\mathfrak{p}_{K}\right)} K_{-d \chi}$ becomes isomorphic to the direct sum of the modules

$$
U\left(\tilde{\mathfrak{g}}_{K}\right) \otimes_{U\left(\tilde{\mathfrak{p}}_{K}\right)} K_{-d \chi_{\sigma}} .
$$

(ii) The $U(\mathfrak{g}) \otimes_{L} K$-module $U\left(\mathfrak{g}_{K}\right) \otimes_{U\left(\mathfrak{p}_{K}\right)} K_{-d \chi}$ is irreducible if and only if for every $\sigma \in \Gamma$ the $U(\tilde{\mathfrak{g}})$-module $U(\tilde{\mathfrak{g}}) \otimes_{U(\tilde{\mathfrak{p}})} K_{-d \chi_{\sigma}}$ is irreducible. If the latter condition is fulfilled, then $\operatorname{Ind}_{\mathbf{P}}^{\mathbf{G}}\left(K_{\chi}\right)$ is a topologically irreducible representation.

Example 4.2.2. With the notation introduced above we let $\tilde{\mathfrak{g}}=\operatorname{Lie}\left(\mathrm{GL}_{2, \tilde{L}}\right)$ and hence we consider $\mathbf{G}=G_{2}(\tilde{L})$ as a locally $L$-analytic group. Let $\chi$ be a locally $L$-analytic character of

$$
\mathbf{T}=\left\{\left(\begin{array}{cc}
t_{1} & 0 \\
0 & t_{2}
\end{array}\right) \mid t_{1}, t_{2} \in \tilde{L}^{*}\right\} .
$$

For $t_{1}, t_{2} \in \tilde{L}^{*}$ sufficiently close to 1 we can write

$$
\chi\left(\begin{array}{cc}
t_{1} & 0 \\
0 & t_{2}
\end{array}\right)=\prod_{\sigma \in \Gamma} \sigma\left(t_{1}\right)^{c_{1, \sigma}} \sigma\left(t_{2}\right)^{c_{2, \sigma}} .
$$

Then the representation $\operatorname{Ind}_{\mathbf{P}}^{\mathbf{G}}\left(K_{\chi}\right)$ is topologically irreducible if for all $\sigma \in \Gamma$,

$$
-\left(c_{1, \sigma}-c_{2, \sigma}\right) \notin \mathbb{Z}_{\geq 0} .
$$

\section{Appendix: Completed Distributions Rings WHICH ARE INTEGRAL DOMAINS}

In this section we consider $L$-uniform groups $H$ as defined in Remark 2.2.5 and we show that there is a sequence $r_{m} \in\left(\frac{1}{p}, 1\right) \cap p^{\mathbb{Q}}$, which tends to 1 , such that the completed distribution rings $D_{r_{m}}(H, K)$ are integral domains.

5.1. The case $H=\mathfrak{o}_{L}$. We begin by considering the case when $H=\mathfrak{o}_{L}$. We denote by $q$ the cardinality of the residue field of $L$, and by $e$ the ramification index of $L$ over $\mathbb{Q}_{p}$. Fix a basis $\mathbb{Z}_{p}$-basis $v_{1}=1, \ldots, v_{n}$ of $\mathfrak{o}_{L}$. Denote by $h_{i}=\delta_{v_{i}}$ the corresponding delta distribution in $D\left(\mathfrak{o}_{L}, K\right)$. For given $m \in \mathbb{Z}_{\geq 0}$ we denote by $\mathcal{F}_{m}\left(\mathfrak{o}_{L}, \mathbb{C}_{p}\right)$ the space of all maps $\mathfrak{o}_{L} \rightarrow \mathbb{C}_{p}$ which are $L$-rigid-analytic on the cosets of $p^{m} \mathfrak{o}_{L}$.

Proposition 5.1.1. (i) Suppose $s \in\left(\frac{1}{p}, 1\right) \cap p^{\mathbb{Q}_{p}}$ has the property that $p^{-1 /(p-1)-1 / e}$ $\leq s^{\varepsilon_{p}}<p^{-1 /(p-1)}$. Then there is a unique continuous $K$-linear map

$$
\phi_{s}: D_{s}\left(\mathfrak{o}_{L}, K\right) \longrightarrow \mathcal{F}_{0}\left(\mathfrak{o}_{L}, \mathbb{C}_{p}\right)_{b}^{\prime}=\operatorname{Hom}_{K}^{\text {cont }}\left(\mathcal{F}_{0}\left(\mathfrak{o}_{L}, \mathbb{C}_{p}\right), \mathbb{C}_{p}\right)_{b}
$$

which makes the diagram

$$
\begin{array}{ccc}
D_{s}\left(\mathfrak{o}_{L}, K\right) & \stackrel{\phi_{s}}{\longrightarrow} & \mathcal{F}_{0}\left(\mathfrak{o}_{L}, \mathbb{C}_{p}\right)_{b}^{\prime} \\
D\left(\mathfrak{o}_{L}, K\right) & \longrightarrow & C^{a n}\left(\mathfrak{o}_{L}, \mathbb{C}_{p}\right)_{b}^{\prime}
\end{array}
$$

commutative. The vertical maps being the canonical ones. Moreover, $\phi_{s}$ is injective. 
(ii) Suppose $r \in\left(\frac{1}{p}, 1\right) \cap p^{\mathbb{Q}}$ is such that, for some $m \in \mathbb{N}_{0}$, the number $s=r^{p^{m}}$ has the property that $s>\frac{1}{p}$ and $p^{-1 /(p-1)-1 / e q^{e m}} \leq s^{\varepsilon_{p}}<p^{-1 /(p-1)}$. Then there is a unique continuous $K$-linear map

$$
\phi_{r}: D_{r}\left(\mathfrak{o}_{L}, K\right) \longrightarrow \mathcal{F}_{m}\left(\mathfrak{o}_{L}, \mathbb{C}_{p}\right)_{b}^{\prime}=\operatorname{Hom}_{K}^{\text {cont }}\left(\mathcal{F}_{m}\left(\mathfrak{o}_{L}, \mathbb{C}_{p}\right), \mathbb{C}_{p}\right)_{b}
$$

which makes the diagram

$$
\begin{array}{ccc}
D_{s}\left(\mathfrak{o}_{L}, K\right) & \stackrel{\phi_{s}}{\longrightarrow} & \mathcal{F}_{0}\left(\mathfrak{o}_{L}, \mathbb{C}_{p}\right)_{b}^{\prime} \\
\uparrow & & \multirow{\jmath}{\uparrow}{} \\
D_{r}\left(\mathfrak{o}_{L}, K\right) & \stackrel{\phi_{r}}{\longrightarrow} & \mathcal{F}_{m}\left(\mathfrak{o}_{L}, \mathbb{C}_{p}\right)_{b}^{\prime}
\end{array}
$$

commutative. The vertical maps being the canonical ones. Moreover, $\phi_{r}$ is injective. In particular, the canonical map $D_{r}\left(\mathfrak{o}_{L}, K\right) \rightarrow D_{s}\left(\mathfrak{o}_{L}, K\right)$ on the left of the diagram is injective.

Proof. Step 1. Before proving (i) and (ii) we will carry out some preliminary considerations related to functions in $\mathcal{F}_{m}\left(\mathfrak{o}_{L}, \mathbb{C}_{p}\right)$. To do this we will heavily rely on the paper [ST4], especially sections 3 and 4. By [ST4], Proposition 4.5, any function $f \in \mathcal{F}_{m}\left(\mathfrak{o}_{L}, \mathbb{C}_{p}\right)$ has a generalized Mahler expansion of the form

$$
f(z)=\sum_{\beta \geq 0} c_{\beta} P_{\beta}(z \Omega)
$$

with $\left|c_{\beta}\right| p^{\beta / e q^{e m-1}(q-1)} \rightarrow 0$. The polynomials $P_{\beta}(Y)$ are those defined in ST4, Def. 4.1. $\Omega=\Omega_{t_{0}^{\prime}}$ is the period of the Lubin-Tate group $\mathcal{G}$ in ST4. Consider a distribution of the form

$$
\mathbf{b}_{(m)}^{\gamma}:=\left(h_{1}^{p^{m}}-1\right)^{\gamma} .
$$

By [ST4, Lemma 4.6 (2), we have

$$
\mathbf{b}_{(m)}^{\gamma}\left(P_{\beta}(z \Omega)\right)=\left\{\left(F_{p^{m}} t_{0}^{\prime}\right)^{\gamma}, P_{\beta}(-\cdot \Omega)\right\}
$$

and by (9) of that lemma we have

$$
\left\{\left(F_{p^{m} t_{0}^{\prime}}\right)^{k}, P_{\ell}(-\cdot \Omega)\right\}=\frac{1}{\ell !} \frac{d^{\ell}\left(F_{p^{m}} t_{0}^{\prime}\right)^{k}}{d Z^{\ell}}(0),
$$

where the power series $F_{a t_{0}^{\prime}}(Z) \in \mathfrak{o}_{\mathbb{C}_{p}}[[Z]]$ is defined in section 3 of [ST4]. It follows from the formula before Proposition 3.1 in [ST4] that

$$
F_{p^{m}} t_{0}^{\prime}(Z)=F_{t_{0}^{\prime}}\left(\left[p^{m}\right]_{\mathcal{G}}(Z)\right) \text {. }
$$

Put $\rho_{m}=p^{-1 / e q^{e m}}(q-1)$. By $\left[\mathrm{ST} 4\right.$, Lemma 3.2, the map $\left[p^{m}\right]_{\mathcal{G}} \operatorname{maps} \mathbf{B}\left(\rho_{m}\right)$ to $\mathbf{B}\left(\rho_{0}\right)$. Here, $\mathbf{B}(\rho)$ denotes the affinoid disk of radius $\rho$. As $\rho_{0}=p^{-1 / e(q-1)}$ we deduce from ST4], Lemma 3.4 (c), and the proof given there, that $F_{t_{0}^{\prime}}$ maps $\mathbf{B}\left(\rho_{0}\right)$ into $\mathbf{B}\left(\rho_{0}\left|\Omega_{t_{0}^{\prime}}\right|\right)=\mathbf{B}\left(p^{-1 /(p-1)}\right)$. This implies that

$$
|Z| \leq \rho_{m} \Rightarrow\left|F_{p^{m}} t_{0}^{\prime}(Z)\right| \leq p^{-1 /(p-1)}
$$

Next we write

$$
F_{p^{m} t_{0}^{\prime}}(Z)=\lambda_{1} Z+\lambda_{2} Z^{2}+\lambda_{3} Z^{3}+\ldots \in \mathfrak{o}_{\mathbb{C}_{p}}[[Z]]
$$


It follows from (5.1.2) that $\left|\lambda_{\ell}\right| \rho_{m}^{\ell} \leq p^{-1 /(p-1)}$ for all $\ell \geq 1$, i.e.,

$$
\left|\lambda_{\ell}\right| \leq p^{-\frac{1}{p-1}+\frac{\ell}{e q^{e m}(q-1)}} .
$$

Write

$$
F_{p^{m} t_{0}^{\prime}}(Z)^{k}=\sum_{\ell \geq k}\left(\sum_{\substack{\ell_{1}+\ldots+\ell_{k}=\ell \\ \ell_{i} \geq 1}} \lambda_{\ell_{1}} \cdot \ldots \cdot \lambda_{\ell_{k}}\right) Z^{\ell}=\sum_{\ell \geq 0} \lambda_{k, \ell} Z^{\ell} .
$$

Note that $\lambda_{k, \ell}$ vanishes for $k>\ell$. Using (5.1.3) this shows that

$$
\left|\left\{\left(F_{p^{m}} t_{0}^{\prime}\right)^{k}, P_{\ell}(-\cdot \Omega)\right\}\right|=\left|\lambda_{k, \ell}\right| \leq p^{-\frac{k}{p-1}+\frac{\ell}{e q^{e m}(q-1)}} .
$$

Next we consider a (formal) sum of distributions

$$
\mu=\sum_{\gamma \geq 0} d_{\gamma} \mathbf{b}_{(m)}^{\gamma}
$$

with $\left|d_{\gamma}\right| s^{\varepsilon_{p} \gamma} \rightarrow 0$ as $\gamma \rightarrow \infty$. Applying $\mu$ to a function

$$
f=\sum_{\beta \geq 0} c_{\beta} P_{\beta}(z \Omega) \in \mathcal{F}_{m}\left(\mathfrak{o}_{L}, \mathbb{C}_{p}\right)
$$

gives the formal sum

$$
\mu(f)=\sum_{\beta \geq 0, \gamma \geq 0} d_{\gamma} c_{\beta}\left\{\left(F_{p^{m}} t_{0}^{\prime}\right)^{\gamma}, P_{\beta}(-\cdot \Omega)\right\} .
$$

As we have seen above, the term

$$
d_{\gamma} c_{\beta}\left\{\left(F_{p^{m}} t_{0}^{\prime}\right)^{\gamma}, P_{\beta}(-\cdot \Omega)\right\}
$$

vanishes if $\gamma>\beta$. In particular, we only need to consider the case when $\gamma \leq \beta$. It follows from (5.1.4) that the absolute value of (5.1.6) can be bounded from above as follows:

$$
\begin{aligned}
& \left|d_{\gamma} c_{\beta}\left\{\left(F_{p^{m}} t_{0}^{\prime}\right)^{\gamma}, P_{\beta}(-\cdot \Omega)\right\}\right| \\
\leq & \left|d_{\gamma}\right|\left|c_{\beta}\right| p^{-\frac{\gamma}{p-1}+\frac{\beta}{e q^{e m}(q-1)}} \\
= & \left(\left|d_{\gamma}\right| s^{\varepsilon_{p} \gamma}\right) \cdot\left(\left|c_{\beta}\right| p^{\frac{\beta q}{e q^{e m}(q-1)}}\right) \cdot\left(\frac{p^{-1 /(p-1)}}{s^{\varepsilon} p}\right)^{\gamma} \cdot p^{-\frac{\beta}{e q^{e m}}} \\
\leq & \left(\left|d_{\gamma}\right| s^{\varepsilon_{p} \gamma}\right) \cdot\left(\left|c_{\beta}\right| p^{\frac{\beta q}{e q^{e m}(q-1)}}\right) \cdot\left(\frac{p^{-1 /(p-1)}}{s^{\varepsilon} p}\right)^{\beta} \cdot p^{-\frac{\beta}{e q^{e m}}} \\
= & \left(\left|d_{\gamma}\right| s^{\varepsilon_{p} \gamma}\right) \cdot\left(\left|c_{\beta}\right| p^{\frac{\beta q}{e q^{e m}(q-1)}}\right) \cdot\left(\frac{p^{-1 /(p-1)-1 / e q^{e m}}}{s^{\varepsilon p}}\right)^{\beta} .
\end{aligned}
$$

By our assumptions on $d_{\gamma}, c_{\beta}$, and because $p^{-1 /(p-1)-1 / e q^{e m}} \leq s^{\varepsilon_{p}}$, we see that the sum (5.1.5) converges.

Step 2: proof of (i). We apply [Sch, Proposition 5.9, to the ring $D_{s}\left(\mathfrak{o}_{L}, K\right)$. Hence we know that any $\mu \in D_{s}\left(\mathfrak{o}_{L}, K\right)$ has a unique expansion

$$
\mu=\sum_{\gamma \geq 0} d_{\gamma} \mathbf{b}^{\gamma}
$$


where

$$
\mathbf{b}^{\gamma}=\left(h_{1}-1\right)^{\gamma}
$$

with $\left|d_{\gamma}\right| s^{\varepsilon_{p} \gamma} \rightarrow 0$, and the norm $\|\mu\|_{s}$ can be calculated as

$$
\|\mu\|_{s}=\sup _{\gamma}\left|d_{\gamma}\right| s^{\varepsilon_{p} \gamma} .
$$

From step 1 (with $m=0$ ) we know that any such $\mu$ defines a linear form $\phi_{s}(\mu)$ on $\mathcal{F}_{0}\left(\mathfrak{o}_{L}, \mathbb{C}_{p}\right)$ which is obviously continuous. Because

$$
\mathbf{b}^{\gamma}\left(\left(\begin{array}{l}
z \\
\beta
\end{array}\right)\right)= \begin{cases}1, & \gamma=\delta \\
0, & \gamma \neq \delta\end{cases}
$$

the map $D_{s}\left(\mathfrak{o}_{L}, K\right) \rightarrow \mathcal{F}_{0}\left(\mathfrak{o}_{L}, \mathbb{C}_{p}\right)^{\prime}$ is injective. We deduce from (5.1.5) and (5.1.7) that $\left|\phi_{s}(\mu)(f)\right| \leq\|\mu\|_{s}\|f\|$. This proves that $\phi_{s}$ is continuous. Further $\phi_{s}$ is uniquely determined by the commutativity of the diagram, because the image of $D\left(\mathfrak{o}_{L}, K\right)$ in $D_{s}\left(\mathfrak{o}_{L}, K\right)$ is dense. That the diagram is commutative follows from the fact that the group algebra $K\left[\mathfrak{o}_{L}\right]$ is dense in $D\left(\mathfrak{o}_{L}, K\right)$, and because any element $h_{i}-1$ has a convergent expansion in $D_{s}\left(\mathfrak{o}_{L}, K\right)$,

$$
h_{i}-1={ }^{\prime \prime}\left(\left(h_{1}-1\right)+1\right)^{v_{i}}-1^{\prime \prime}=\sum_{\nu=1}^{\infty}\left(\begin{array}{l}
v_{i} \\
\nu
\end{array}\right)\left(h_{1}-1\right)^{\nu} \text {. }
$$

For a function $f$ which is a polynomial of the form $\left(\begin{array}{c}z \\ \beta\end{array}\right)$, it follows from (5.1.8) that

$$
\phi_{s}\left(h_{i}-1\right)(f)=\phi_{s}\left(\sum_{\nu=1}^{\infty}\left(\begin{array}{l}
v_{i} \\
\nu
\end{array}\right)\left(h_{1}-1\right)^{\nu}\right)(f)=f\left(v_{i}\right)-f(0) .
$$

Approximating any $L$-rigid analytic function $f$ on $\mathfrak{o}_{L}$ by polynomials, this holds true for all elements of $\mathcal{F}_{0}\left(\mathfrak{o}_{L}, \mathbb{C}_{p}\right)$. This proves the first assertion.

Step 3: proof of (ii). The subgroup $p^{m} \mathfrak{o}_{L} \subset \mathfrak{o}_{L}$ is uniform pro- $p$, and we can thus consider the canonical $p$-valuation on this subgroup, and the associated norm $\|\cdot\|_{s}$ which we denote for clarity by $\|\cdot\|_{s}^{(m)}$. By [Sch], Lemma 7.4 , the norm $\|\cdot\|_{r}$ on $D\left(\mathfrak{o}_{L}, K\right)$, when restricted to $D\left(p^{m} \mathfrak{o}_{L}, K\right) \subset D\left(\mathfrak{o}_{L}, K\right)$ induces the same topology on $D\left(p^{m} \mathfrak{o}_{L}, K\right)$ as the norm $\|\cdot\|_{s}^{(m)}$. Furthermore, $D_{r}\left(\mathfrak{o}_{L}, K\right)$ is a finite and free (left or right) module over the subring $D_{s}\left(p^{m} \mathfrak{o}_{L}, K\right)$, on a basis of any set $\mathcal{R}$ of coset representatives for $\mathfrak{o}_{L} / p^{m} \mathfrak{o}_{L}$ :

$$
D_{r}\left(\mathfrak{o}_{L}, K\right)=\bigoplus_{a \in \mathcal{R}}\left(\delta_{a}\right)_{r} D_{s}\left(p^{m} \mathfrak{o}_{L}, K\right)
$$

Here, $\left(\delta_{a}\right)_{r}$ denotes the image of the delta distribution $\delta_{a} \in D\left(\mathfrak{o}_{L}, K\right)$ in $D_{r}\left(\mathfrak{o}_{L}, K\right)$. Because of our assumptions on $s$ we can apply $\mathrm{Sch}$, Proposition 5.9, which tells us that any element $\mu \in D_{s}\left(p^{m} \mathfrak{o}_{L}, K\right)$ has a unique expansion

$$
\mu=\sum_{\gamma \geq 0} d_{\gamma} \mathbf{b}_{(m)}^{\gamma}
$$

where

$$
\mathbf{b}_{(m)}^{\gamma}=\left(h_{1}^{p^{m}}-1\right)^{\gamma}
$$

and the norm $\|\mu\|_{s}^{(m)}$ can be calculated as

$$
\|\mu\|_{s}^{(m)}=\sup _{\gamma}\left|d_{\gamma}\right| s^{\varepsilon_{p} \gamma}
$$


As we have seen in step 1 , any $\mu \in D_{s}\left(p^{m} \mathfrak{o}_{L}, K\right)$ induces a continuous linear form $\phi_{r}(\mu)$ on $\mathcal{F}_{m}\left(\mathfrak{o}_{L}, \mathbb{C}_{p}\right)$. By step 2 we have $\phi\left(\delta_{a}\right)(f)=f(a)$ for any $a \in p^{m} \mathfrak{o}_{L}$ and $f \in \mathcal{F}_{m}\left(\mathfrak{o}_{L}, \mathbb{C}_{p}\right)$. In particular, $\phi(\mu)$ vanishes on all functions $f$ which vanish identically on $p^{m} \mathfrak{o}_{L}$, because the group ring $K\left[p^{m} \mathfrak{o}_{L}\right]$ is dense in $D_{s}\left(p^{m} \mathfrak{o}_{L}, K\right)$. For a product $\left(\delta_{a}\right)_{r} \mu$ with $a \in \mathcal{R}$ and $\mu \in D_{s}\left(p^{m} \mathfrak{o}_{L}, K\right) \subset D_{r}\left(\mathfrak{o}_{L}, K\right)$, and $f \in \mathcal{F}_{m}\left(\mathfrak{o}_{L}, \mathbb{C}_{p}\right)$ define

$$
\left(\left(\delta_{a}\right)_{r} \mu\right)(f)=\mu\left[\mathfrak{o}_{L} \rightarrow \mathbb{C}_{p}, z \mapsto f(a+z)\right]
$$

Of course, for any $a \in \mathfrak{o}_{L}$ and any $f \in \mathcal{F}_{m}\left(\mathfrak{o}_{L}, \mathbb{C}_{p}\right)$, the function $z \mapsto f(a+z)$ is again in $\mathcal{F}_{m}\left(\mathfrak{o}_{L}, \mathbb{C}_{p}\right)$. Thus we have a well-defined map

$$
\phi_{r}: D_{r}\left(\mathfrak{o}_{L}, K\right)=\bigoplus_{a \in \mathcal{R}}\left(\delta_{a}\right)_{r} D_{s}\left(p^{m} \mathfrak{o}_{L}, K\right) \longrightarrow \mathcal{F}_{m}\left(\mathfrak{o}_{L}, \mathbb{C}_{p}\right)_{b}^{\prime}
$$

That this map is continuous is easily seen. To see that it is injective, fix $a_{0} \in \mathcal{R}$, and consider the subspace of those $f \in \mathcal{F}_{m}\left(\mathfrak{o}_{L}, \mathbb{C}_{p}\right)$ which vanish outside of $a_{0}+p^{m} \mathfrak{o}_{L}$. Then, if an element

$$
\lambda=\sum_{a \in \mathcal{R}}\left(\delta_{a}\right)_{r} \mu_{a} \in D_{r}\left(\mathfrak{o}_{L}, K\right),
$$

with all $\mu_{a} \in D_{s}\left(p^{m} \mathfrak{o}_{L}, K\right)$, is such that $\phi_{r}(\lambda)=0$, then, for $f$ in the subspace just mentioned,

$$
0=\sum_{a \in \mathcal{R}} \mu_{a}[z \mapsto f(a+z)]=\mu_{a_{0}}\left[z \mapsto f\left(a_{0}+z\right)\right] .
$$

But this means that $\mu_{a_{0}}$ vanishes on all $L$-rigid-analytic functions supported on $p^{m} \mathfrak{o}_{L}$. Because $\phi_{s}$ for $p^{m} \mathfrak{o}_{L}$ is injective (by step 2), this means that $\mu_{a_{0}}=0$. Therefore, $\phi_{r}$ is injective as well.

\subsection{Reduction to the case $H=\mathfrak{o}_{L}$.}

Proposition 5.2.1. Let $H$ be a locally L-analytic group which is L-uniform (cf. Remark 2.2.5). We define the norms $\bar{q}_{r}$ using the canonical p-valuation on $H$ (cf. 2.2.6).

(i) Let $r \in\left(\frac{1}{p}\right) \cap p^{\mathbb{Q}}$ and $s=r^{p^{m}}$ be as in Proposition 5.1.1 (ii). Then the canonical map $D_{r}(H, K) \rightarrow D_{s}(H, K)$ is injective.

(ii) There is a sequence of numbers $r_{m} \in\left(\frac{1}{p}, 1\right) \cap p^{\mathbb{Q}}$, which tends to 1 , such that the completed distribution rings $D_{r_{m}}(H, K)$ are integral domains.

Proof. (i) Let $\Lambda$ be as in Lemma 2.2.4 By DDMS, 9.10, we see that $\Lambda$ is a uniform $\mathbb{Z}_{p}$-Lie algebra: $[\Lambda, \Lambda] \subset p^{\varepsilon_{p}} \Lambda$. By [DDMS], 9.8, the Baker-Campbell-Hausdorff series $\Phi(X, Y)=\exp _{H}^{-1}\left(\exp _{H}(X) \exp _{H}(Y)\right)$ converges on $\Lambda \times \Lambda$ and maps $\Lambda \times \Lambda$ into $\Lambda$. In fact, we can identify $H=\exp _{H}(\Lambda)$ with $\Lambda$, the group multiplication being given by $\Phi(\cdot, \cdot)$. The members of the lower $p$-series are then $\exp _{H}\left(p^{k} \Lambda\right), k \geq 0$. Let $\left(v_{1}, \ldots, v_{n}\right)$ be a $\mathbb{Z}_{p}$-basis of $\mathfrak{o}_{L}$, and let $\left(\mathfrak{x}_{j}\right)_{j=1}^{d}$ be an $\mathfrak{o}_{L}$-basis of $\Lambda$. Then $\left(v_{i} \mathfrak{x}_{j}\right)_{1 \leq i \leq n, 1 \leq j \leq d}$ is a $\mathbb{Z}_{p}$-basis for $\Lambda$. The map $\psi: \mathfrak{o}_{L}^{d} \stackrel{\sim}{\longrightarrow} H$ defined by sending

$$
\left(z_{j}\right)_{j=1}^{d}=\left(a_{1, j} v_{1}+\ldots+a_{n, j} v_{n}\right)_{j=1}^{d} \in \mathfrak{o}_{L}^{d}
$$

to

$$
\begin{aligned}
& \exp _{H}\left(v_{1} \mathfrak{x}_{1}\right)^{a_{1,1}} \cdot \ldots \cdot \exp _{H}\left(v_{n} \mathfrak{x}_{1}\right)^{a_{n, 1}} \cdot \ldots \cdot \exp _{H}\left(v_{1} \mathfrak{x}_{d}\right)^{a_{1, d}} \cdot \ldots \cdot \exp _{H}\left(v_{n} \mathfrak{x}_{d}\right)^{a_{n, d}} \\
& =\exp _{H}\left(z_{1} \mathfrak{x}_{1}\right) \cdot \ldots \cdot \exp _{H}\left(z_{d} \mathfrak{x}_{d}\right)
\end{aligned}
$$


is then a homeomorphism, cf. Lemma 2.2.4 (3). It is shown in [ST3, Proposition A.3, that

$$
D\left(\mathfrak{o}_{L}^{d}, K\right)=D\left(\mathfrak{o}_{L}, K\right) \widehat{\otimes}_{K, \pi} \ldots \widehat{\otimes}_{K, \pi} D\left(\mathfrak{o}_{L}, K\right)
$$

(with $d$ factors on the right). Here, the completed topological tensor product is the completion of the ordinary tensor product with respect to the projective tensor product topology (indicated by $\pi$ ). In [ST3], Proposition A.3, it is the inductive tensor product topology that is used. However, by $[\underline{S}$, 17.6, the inductive and the projective tensor product topologies coincide in this case, as the distribution algebras are Fréchet spaces. Using the homeomorphism $\psi$ we get an isomorphism of topological vector spaces

$$
\psi^{*}: C_{L}^{a n}(H, K) \stackrel{\simeq}{\longrightarrow} C_{L}^{a n}\left(\mathfrak{o}_{L}^{d}, K\right)
$$

which gives, after dualizing, an isomorphism of topological vector spaces

$$
\left(\psi^{*}\right)^{\prime}: D\left(\mathfrak{o}_{L}, K\right) \widehat{\otimes}_{K, \pi} \ldots \widehat{\otimes}_{K, \pi} D\left(\mathfrak{o}_{L}, K\right)=D\left(\mathfrak{o}_{L}^{d}, K\right) \stackrel{\simeq}{\longrightarrow} D(H, K) .
$$

Tracing through the definition of the norms $\bar{q}_{r}$ on $D(H, K)$ as constructed in 2.2 .6 shows that $\bar{q}_{r}$ corresponds to the norm $\bar{q}_{r}^{\mathfrak{o}_{L}} \otimes \ldots \otimes \bar{q}_{r}^{\mathfrak{o}_{L}}$ on

$$
D\left(\mathfrak{o}_{L}, K\right) \widehat{\otimes}_{K, \pi} \ldots \widehat{\otimes}_{K, \pi} D\left(\mathfrak{o}_{L}, K\right)
$$

induced by the norms $\bar{q}_{r}^{\mathfrak{o}_{L}}$ on each factor $D\left(\mathfrak{o}_{L}, K\right)$. Here again, the norms $\bar{q}_{r}^{\mathfrak{o}_{L}}$ are defined as in 2.2.6. using the canonical $p$-valuation on the uniform group $\mathfrak{o}_{L}$. The completion of

$$
D\left(\mathfrak{o}_{L}, K\right) \widehat{\otimes}_{K, \pi} \ldots \widehat{\otimes}_{K, \pi} D\left(\mathfrak{o}_{L}, K\right)
$$

with respect to the induced norm $\bar{q}_{r}^{\mathfrak{o}_{L}} \otimes \ldots \otimes \bar{q}_{r}^{\mathrm{o}_{L}}$ is then

$$
D_{r}\left(\mathfrak{o}_{L}, K\right) \widehat{\otimes}_{K, \pi} \ldots \widehat{\otimes}_{K, \pi} D_{r}\left(\mathfrak{o}_{L}, K\right)
$$

It follows from Em, 1.1.27, that if the map $D_{r}\left(\mathfrak{o}_{L}, K\right) \rightarrow D_{s}\left(\mathfrak{o}_{L}, K\right)$ is injective, then so is the (iterated) tensor product of this map with itself. By Proposition 5.1.1 we know that this map is injective if $r$ and $s$ are in relation as stated in Proposition 5.1.1. Therefore we have proved that the canonical map $D_{r}(H, K) \rightarrow D_{s}(H, K)$ is injective (for $r$ and $s$ as in Proposition 5.1.1).

(ii) It follows from $\left[\mathrm{Sch}\right.$, Proposition 5.6, that if $s>\frac{1}{p}$ and $s^{\varepsilon_{p}}<p^{-1 /(p-1)}$, then $D_{s}(H, K)$ is an integral domain. Hence, $D_{r}(H, K)$ is an integral domain. It is easily seen that one can find an increasing sequence of numbers $r_{m} \in\left(\frac{1}{p}, 1\right) \cap p^{\mathbb{Q}}$ which tends to 1 , and such that $s_{m}=r_{m}^{p^{m}}$ has the property that $s_{m}>\frac{1}{p}$ and $p^{-1 /(p-1)-1 / e q^{e m}} \leq s_{m}^{\varepsilon_{p}}<p^{-1 /(p-1)}$.

\section{ACKNOWLEDGEMENTS}

We would like to thank Tobias Schmidt and Jan Kohlhaase for helpful discussions on distribution algebras. We thank the SFB 478 "Geometrische Strukturen in der Mathematik" at Münster for financial support of travel expenses. Finally, we thank the referee for his careful reading, in particular, for pointing out a gap in a previous version. 


\section{REFERENCES}

[BGR] S. Bosch, U. Güntzer, R. Remmert, Non-Archimedean Analysis. Grundlehren der Mathematischen Wissenschaften, 261. Springer-Verlag, Berlin (1984). MR746961|(86b:32031)

[BS] C. Breuil, P. Schneider, First steps towards p-adic Langlands Functoriality, J. Reine Angew. Math. 610, 149-180 (2007) MR2359853 (2009f:11147)

[BT1] F. Bruhat, J. Tits, Groupes réductifs sur un corps local: I. Données radicielles valuées., Publ. Math. Inst. Hautes Étud. Sci. 41, 5-251 (1972). MR0327923 (48:6265)

[BT2] F. Bruhat, J. Tits, Groupes réductifs sur un corps local: II. Schémas en groupes. Existence d'une donnée radicielle valuée., Publ. Math., Inst. Hautes Étud. Sci. 60, 197-376 (1984). MR0756316 (86c:20042)

[Ca] P. Cartier, Representations of $\mathfrak{p}$-adic groups: a survey. Automorphic forms, representations and $L$-functions (Proc. Sympos. Pure Math., Oregon State Univ., Corvallis, Ore., 1977), Part 1, pp. 111-155, Proc. Sympos. Pure Math., 33, Amer. Math. Soc., Providence, R.I. (1979). MR546593 (81e:22029)

[D] J. Dixmier, Enveloping Algebras. North-Holland Mathematical Library, North-Holland Publishing Co., Amsterdam, New York, Oxford (1977). MR0498740(58:16803b)

[DDMS] J. D. Dixon, M. P. F. du Sautoy, A. Mann, D. Segal, Analytic pro-p groups. Second edition. Cambridge Studies in Advanced Mathematics, 61, Cambridge University Press, Cambridge (1999). MR1720368 (2000m:20039)

[Em] M. Emerton, Locally analytic vectors in representations of locally p-adic analytic groups, To appear in Memoirs of the AMS.

[Fe] C. T. Feaux de Lacroix, Einige Resultate über die topologischen Darstellungen p-adischer Liegruppen auf unendlich dimensionalen Vektorräumen über einem p-adischen Körper, Schriftenreihe des Mathematischen Instituts der Universität Münster, 3. Serie, Heft 23 (1999). MR 1691735 (2000k:22021)

[Fr] H. Frommer, The locally analytic principal series of split reductive groups, Preprintreihe SFB 478, Münster, Heft 265 (2003); available at http://3dsp.unimuenster.de/wwwmath.uni-muenster.de/sfb/about/publ/frommer01.html.

[K1] J. Kohlhaase, Invariant distributions on p-adic analytic groups, Duke Math. Journal, vol. 137, no. 1, 19-62 (2007). MR2309143 (2008j:22024)

[K2] J. Kohlhaase, The cohomology of locally analytic representations, preprint, Münster (2008); available at: http://wwwmath.uni-muenster.de/sfb/about/publ/kohlhaase.html, to appear in J. Reine Angew. Math. (Crelle).

[KS] M. Kisin, M. Strauch, Locally analytic cuspidal representations of $G L_{2}$ and related groups. Journal of the Institute of Mathematics of Jussieu, 5 (3), 373-421 (2006). MR2241928 (2007j:22028)

[L] M. Lazard, Groupes analytiques p-adique, Publ. Math., Inst. Hautes Étud. Sci. 26, 5-219 (1965). MR0209286 (35:188)

[Mo] Y. Morita, Analytic representations of $\mathrm{SL}_{2}$ over a $\mathfrak{p}$-adic number field. II., Automorphic forms of several variables (Katata, 1983), 282-297, Progr. Math., 46, Birkhäuser Boston, Boston, MA (1984). MR763019 (86j:22027)

[Sch] T. Schmidt, Auslander Regularity of p-adic Distribution Algebras, Represent. Theory 12, 37-57 (2008). MR2375595(2009b:22018)

[S] P. Schneider, Nonarchimedean functional analysis, Springer Monographs in Mathematics, Springer-Verlag, Berlin (2002). MR1869547(2003a:46106)

[S-S] P. Schneider, U. Stuhler, Representation theory and sheaves on the Bruhat-Tits building, Publ. Math. IHÉS 85, 97-191 (1997). MR1471867(98m:22023)

[ST1] P. Schneider, J. Teitelbaum, Locally analytic distributions and p-adic representation theory, with applications to $\mathrm{GL}_{2}$, J. Amer. Math. Soc. 15, no. 2, 443-468 (2002). MR 1887640 (2003b:11132)

[ST2] P. Schneider, J. Teitelbaum, Algebras of p-adic distributions and admissible representations, Invent. Math. 153, no. 1, 145-196 (2003). MR1990669 (2004g:22015)

[ST3] P. Schneider, J. Teitelbaum, Duality for admissible locally analytic representations, Represent. Theory 9, 297-326 (2005). MR2133762(2006a:22016) 
[ST4] P. Schneider, J. Teitelbaum, p-adic Fourier theory, Doc. Math. 6, 447-481 (2001). MR.1871671 (2002j:11143)

[Ti] J. Tits, Reductive groups over local fields, Automorphic forms, representations and $L$-functions, Proc. Symp. Pure Math. Am. Math. Soc., Corvallis/Oregon 1977, Proc. Symp. Pure Math. 33, 1, 29-69 (1979). MR546588 (80h:20064)

Fachgruppe Mathematik and Informatik, Bergische Universität Wuppertal, Gausstrasse 20, 42097 Wuppertal, Germany

E-mail address: orlik@math.uni-wuppertal.de

Department of Mathematics, Indiana University, 831 East Third Street, BloomingTON, INDIANA 47401

E-mail address: mstrauch@indiana.edu 